\title{
Acute Post-Exercise Recovery Strategies in Cycling: A Review
}

\author{
Ryan Overmayer ${ }^{1}$, Francisco Tavares ${ }^{1}$ and Matthew Driller ${ }^{1} \bowtie$
}

\begin{abstract}
Cycling events often include multiple races a day or racing over consecutive days. Congested competition schedules and increased training load have led to the implementation of recovery strategies; with the goal of alleviating postexercise fatigue and enhancing subsequent performance. This review aims to review the efficacy of recovery strategies used following different cycling events. Compression garments have been shown to improve subsequent 30 s - 30min mean cycling power and 5-min max cycling power, while cold water immersion may improve 5-15s sprint cycling power output, 1-15min time trial (TT) total work performed and mean power output in hot and humid conditions. Cold water immersion was also more beneficial than active recovery at improving total work performed. Contrast water therapy could increase $15 \mathrm{~s}-15 \mathrm{~min}$ TT work performed and sprint mean and peak power output. Similarly, active recovery has been shown to improve low intensity $3-15 \mathrm{~min}$ cycling power and time to completion. Conversely, hot water immersion appears to be detrimental to sprint power output and TT power output over consecutive days. Thermoneutral water immersion appears beneficial for improving average cycling speed and time to completion during a 20-km TT, where humidification therapy and sports massage are beneficial at improving sprint and middle duration time trial performance. A combination of recovery strategies appear more beneficial than stand-alone strategies and various combinations should be explored further.
\end{abstract}

Keywords: fatigue; cyclist; cold water immersion; compression

$\triangle$ Contact email: mdriller@waikato.ac.nz (M Driller)

${ }^{1}$ Faculty of Health, Sport and Human Performance, University of

Waikato, Hamilton, New Zealand

Received: 07 November 2018. Accepted: 28 December 2018.

\section{Introduction}

There are many disciplines in professional cycling such as track cycling, road cycling, mountain biking and bicycle motocross (BMX) (Edwards and Corte 2010; Jeukendrup et al. 2000; Marquet et al. 2015). These disciplines involve multiple races a day or racing over consecutive days (Marquet et al. 2015; Ménétrier et al. 2013; Versey et al. 2011). During training and congested competition schedules, recovery strategies are thought to alleviate post-exercise fatigue and enhance subsequent performance (Argus et al. 2013; Nédélec et al. 2013). Consequently, a substantial challenge is placed on athletes and coaches to ensure optimal recovery is attained, and has been one of the contributing factors for the development of novel recovery strategies to enhance performance (Argus et al. 2013; Stanley et al. 2013). The main purpose of this review is to summarize the scientific literature on acute post-exercise recovery strategies implemented in the sport of cycling.

\section{Liretature Search}

The relevant literature for this review was obtained from a search within Google Scholar, MEDLINE/PubMed, SPORTDiscus, Web of Science and Cochrane databases. Based on a search of these databases, to our knowledge, there is currently no published review examining the literature on recovery strategies used with cyclists as the participants of interest. Included terms for the searches were: "cyclist/cycling" + either: "Recovery strategies", "cold water immersion", "active recovery", "electromyostimulation", "massage recovery", "compression garments recovery", "cryotherapy", "water immersion recovery", "hydrotherapy recovery", "static stretching recovery", "dynamic stretching recovery", "ice". The inclusion criteria was limited to the English language and studies published prior to 2018. Studies which examined cycling but did not use cyclists as subjects were excluded from the review. Studies which examined cycling but did not use cyclists as subjects were excluded from the review. The rationale for this was to provide an accurate representation of the impact of recovery strategies when used in a relevant context for practitioners; with cyclists and in cycling settings. Twenty-seven studies were included for analysis. Recovery strategies examined include active recovery (AR), sports massage (SM), cold water immersion (CWI), compression garments (COMP), electromyostimulation (EMS), humidification therapy (HUM), passive recovery in water (PRW), active recovery in water (ARW), static stretching (SS), contrast water therapy (CWT), compression stockings (CS), hot water immersion/therapy (HWI), cold compression therapy (CCT) thermoneutral water immersion (TWI) and a combination of active recovery and sports massage. 


\section{Fatigue in Cycling}

In order to discuss the potential fatigue mechanisms associated with cycling, one must first determine the duration of the event (Craig and Norton 2001). For example, while the winning time for the men's Omnium flying lap race at the 2016 Rio Olympics was $12.506 \mathrm{~s}$, the winning time for the road race was $6: 10.05 \mathrm{~s}$; resulting in a variance in exercise intensity, energy utilization and associated fatigue (Black et al. 2017; Jeukendrup et al. 2000). Therefore, cycling events have been categorized with race duration (Table 1). The following section provides a general overview of fatigue associated with the category durations provided.

\section{Fatigue During Sprint Cycling}

Humans possess Adenosine Triphosphate (ATP) reserves for $\sim 2$ seconds of maximal contraction (Cramer 2008; Kenney et al. 2015). Since ATP serves as the currency for the production of mechanical work, one can expect that a reduction in ATP leads to a state where the capacity to produce mechanical work is reduced (Kenney et al. 2015). In a brief event such as sprint cycling (i.e. $200 \mathrm{~m}$ track sprint), energy production is highly dependent on the anaerobic system (Jeukendrup et al. 2000). For example, during a $200 \mathrm{~m}$ track sprint, the alactic and anaerobic systems contribute 40 and $55 \%$ of energy production, respectively (Jeukendrup et al. 2000). Therefore, performance decrements in these events have been attributed to a combination of 'peripheral metabolic' and 'central/neural' mechanisms (Craig and Norton 2001; Gardner et al. 2009). Peripheral metabolic mechanisms are associated not only to a breakdown of phosphocreatine (PCr) and a subsequent increase in inorganic phosphates $(\mathrm{Pi})$, but also to a reduction in cross-bridge cycling and force production (Temesi et al. 2017). Neural mechanisms include a reduction of the central nervous system (CNS) to drive motor neurons; therefore decreasing the number of active motor units (MU), including those innervating fast twitch muscle fibers, responsible for maximal force production (Gardner et al. 2009; Phillips 2015). Thus, a reduction in the capability to recruit fast twitch $\mathrm{MU}$, will
Table 1. Men's cycling events categorised according to duration.

\begin{tabular}{|c|c|c|}
\hline Category & Duration & Events \\
\hline Sprint & $0-30 \mathrm{sec}$ & $\begin{array}{l}\text { Track Omnium Flying Lap } \\
(12.51 \mathrm{~s})^{*}\end{array}$ \\
\hline $\begin{array}{l}\text { Short- } \\
\text { duration }\end{array}$ & $\begin{array}{l}30-120 \\
\text { sec }\end{array}$ & $\begin{array}{l}\text { Track Team Sprint } \\
(42.44 \mathrm{~s})^{*} \\
\text { BMX } \\
(34.64 \mathrm{~s})^{*} \\
\text { Track Omnium 1-km TT } \\
(60.92 \mathrm{~s})^{*}\end{array}$ \\
\hline $\begin{array}{l}\text { Middle- } \\
\text { duration }\end{array}$ & $\begin{array}{l}2 \min -30 \\
\min \end{array}$ & $\begin{array}{l}\text { Track Keirin } \\
(2: 27 \text { s submaximal }+34 \text { s sprint })^{*} \\
\text { Track Omnium IP } \\
(4: 14.98 \mathrm{~s})^{*} \\
\text { Track Team Pursuit } \\
(3: 50.27 \mathrm{~s})^{*} \\
\text { Track Omnium Elimination } \\
\text { (Approx. } 13: 49 \mathrm{~s} \text { submaximal with }_{\text {sprint bursts })^{*}} \\
\text { Track Omnium SR } \\
(17: 24 \mathrm{~s})^{*}\end{array}$ \\
\hline Endurance & $\begin{array}{l}\text { Over } 30 \\
\text { min }\end{array}$ & $\begin{array}{l}\text { Track Omnium Points Race } \\
(46: 23 s)^{*} \\
\text { Road Race } \\
(6: 10: 05 s)^{*} \\
\text { Road Individual TT } \\
(1: 12: 15.42 s)^{*} \\
\text { Cross-Country MB } \\
(1: 33: 28 s)^{*}\end{array}$ \\
\hline
\end{tabular}

ultimately result in a reduction of power output during sprint cycling (Gardner et al. 2009).

Fatigue During Short-Duration Cycling

During short-duration events (Table 1), both the anaerobic and aerobic systems contribute to the vast majority of energy production (Jeukendrup et al. 2000). For example, during a female $500 \mathrm{~m}$ cycling sprint (duration $\sim 35 \mathrm{~s}$ ), the anaerobic glycolytic and aerobic contribution is suspected to be $45 \& 35 \%$, respectively (Jeukendrup et al. 2000). Moreover, the anaerobic glycolytic and aerobic contribution during a male $1000 \mathrm{~m}$ track cycling event (duration $\sim 60$ s) is suspected to be 40

Table 2. Pressure exerted by compression garment type and reporting method.

\begin{tabular}{|c|c|c|c|c|}
\hline Author & Garment Type & $\begin{array}{l}\text { Calf } \\
\text { Compression } \\
(\mathrm{mmHg})\end{array}$ & $\begin{array}{l}\text { Thigh } \\
\text { Compression }(\mathrm{mmHg})\end{array}$ & Reporting method \\
\hline Argus et al., 2013 & Full length tights & $27 \pm 6$ & $18 \pm 2$ & Kikuhime \\
\hline $\begin{array}{c}\text { Driller \& Halson, } \\
2013\end{array}$ & Full length tights & $21 \pm 3$ & $12 \pm 3$ & Unpublished observations \\
\hline $\begin{array}{c}\text { Chatard et al., } \\
2004\end{array}$ & $\begin{array}{l}\text { Compression } \\
\text { stockings }\end{array}$ & 18 & 12 & Manufacturer report \\
\hline $\begin{array}{l}\text { Ménétrier et al., } \\
2013\end{array}$ & $\begin{array}{l}\text { Compression } \\
\text { stockings }\end{array}$ & 27 & 14 & Manufacturer report \\
\hline Argus et al., 2013 & Full length tights & $27 \pm 6$ & $18 \pm 2$ & Kikuhime \\
\hline
\end{tabular}


\& 50\%, respectively (Jeukendrup et al. 2000). Conversely, the alactic system is believed to only contribute $10-20 \%$ of total energy production during events of this duration (Jeukendrup et al. 2000). The dependency on the anaerobic glycolytic system is associated with an increase in metabolites and therefore a loss of muscle function (Cairns 2006; Robergs et al. 2004; Westerblad et al. 2002). While traditionally thought that increased $\mathrm{H}^{+}$was the main metabolite which contributed to fatigue (Cairns 2006), Degroot and

Table 3. Summary of studies examining the use of compression garments post-exercise in cyclists.

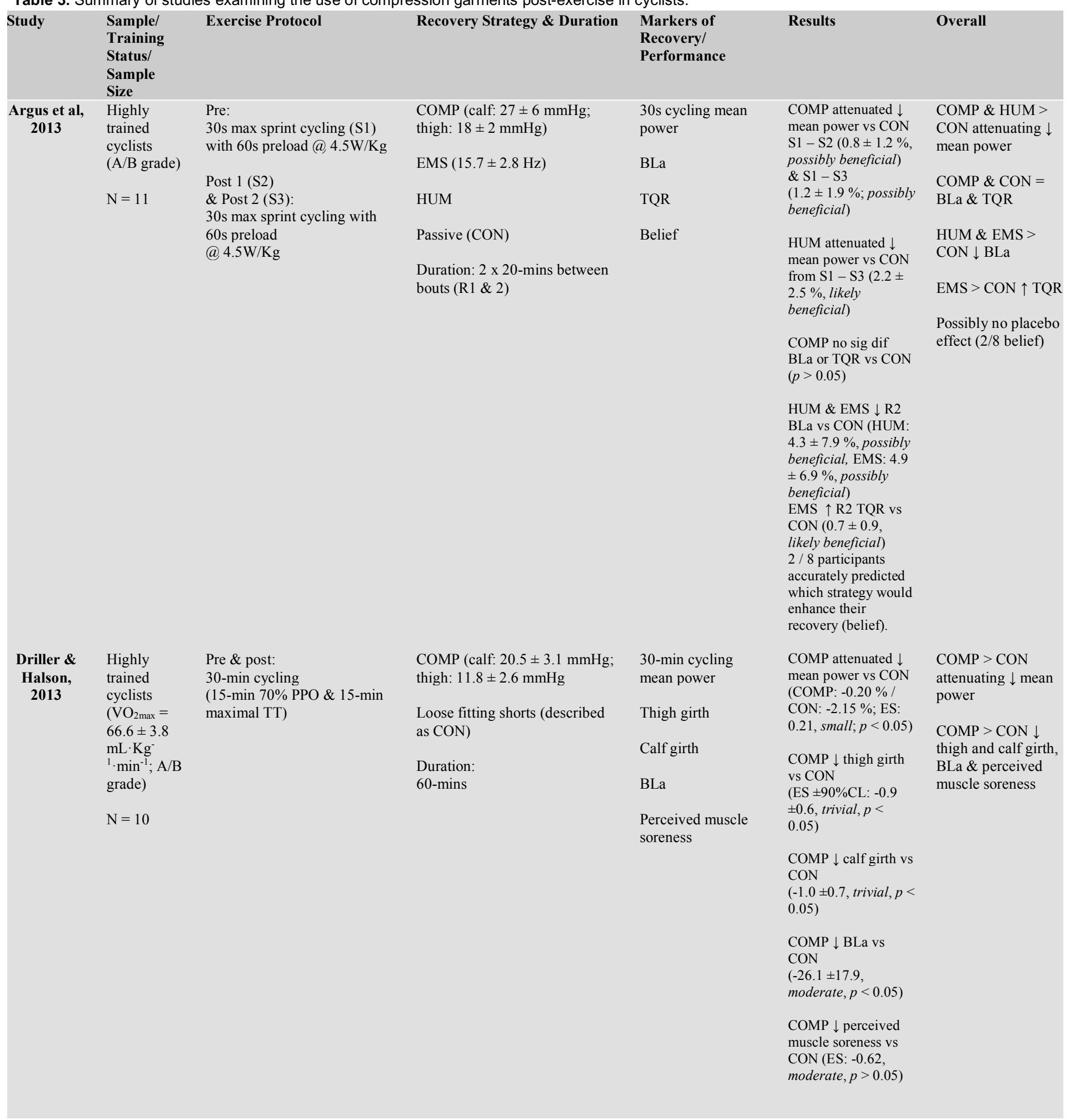




\begin{tabular}{|c|c|c|c|c|c|c|}
\hline $\begin{array}{l}\text { Chatard } \\
\text { et al, } 2004\end{array}$ & $\begin{array}{l}\text { Trained } \\
\text { elderly } \\
\text { cyclists } \\
\left(\mathrm{VO}_{2 \max }=\right. \\
49 \pm 6 \\
\mathrm{~mL} \cdot \mathrm{Kg}^{-} \\
{ }^{1} \cdot \mathrm{min}^{-1} ; \\
\text { mean age } \\
=63 \text { years; } \\
\text { training } \\
\text { years }=10 \\
\pm 4 \text { years }) \\
\mathrm{N}=12\end{array}$ & $\begin{array}{l}\text { Pre \& post: } \\
\text { 5-min max cycling }\end{array}$ & $\begin{array}{l}\text { CS (calf: } 18 \mathrm{mmHg} \text {; thigh: } \\
12 \mathrm{mmHg} \text { ) } \\
\text { Passive without CS (CON) }\end{array}$ & $\begin{array}{l}\text { 5-min cycling } \\
\text { max power } \\
\text { HR } \\
\text { BLa \& hematocrit } \\
\text { RPE }\end{array}$ & $\begin{array}{l}\text { CS attenuated } \downarrow \\
\text { max power vs } \\
\text { CON }(2.1 \pm 1.4 \% \text {, } \\
p<0.01) \\
\text { CS no sig dif for } \\
\text { HR post-recovery } \\
\text { or RPE vs CON ( } p \\
>0.01 \text { ) } \\
\text { CS } \downarrow \text { BLa and } \\
\text { hematocrit during } \\
\text { recovery vs CON } \\
\text { (BLa: } F=7.7 \text {, } \\
\text { haematocrit: } F= \\
6.8, p<0.01 \text { ) }\end{array}$ & $\begin{array}{l}\mathrm{CS}>\mathrm{CON} \\
\text { attenuating } \downarrow \\
\text { max power } \\
\mathrm{CS}>\mathrm{CON} \downarrow \mathrm{BLa} \\
\text { and hematocrit } \\
\text { during recovery } \\
\mathrm{CS} \& \mathrm{CON}= \\
\mathrm{HR} \text {, and RPE }\end{array}$ \\
\hline $\begin{array}{l}\text { Menetrier } \\
\text { et al, } 2013\end{array}$ & $\begin{array}{l}\text { Competitive } \\
\text { male } \\
\text { cyclists } \\
(\mathrm{PPO}=5.0 \\
\pm 0.2 \mathrm{~W} / \mathrm{Kg} \text { ) } \\
\mathrm{N}=12\end{array}$ & $\begin{array}{l}\text { Pre: } \\
\text { 10-min cycling } \\
\text { (5-mins } 80 \% \text { PPO \& 5- } \\
\text { mins } 90 \% \text { PPO } \\
\text { Post: } \\
\text { 5-min maximal cycling }\end{array}$ & $\begin{array}{l}\text { Passive seated [ } 21^{\circ} \mathrm{C} \text {, } \\
\sim 30 \% \text { rh] (CON) } \\
\text { CWT }(4 \times 3 \text {-min to top } \\
\text { thigh; } 1 \text {-min cold bath [10- } \\
\left.12^{\circ} \mathrm{C}\right], 2 \text {-min hot bath [36- } \\
\left.38^{\circ} \mathrm{C}\right], 5 \text { s changeover) } \\
\text { CS (according to } \\
\text { manufacturer: calf }= \\
27 \mathrm{mmHg} \text {; thigh = } \\
14 \mathrm{mmHg}) \\
\text { Duration: } \\
\text { 1.5-mins passive seated } \\
\text { pre and post condition } \\
\text { 12-mins per condition }\end{array}$ & $\begin{array}{l}\text { 5-min maximal } \\
\text { cycling mean } \\
\text { power } \\
\text { BLa } \\
\text { Perceived } \\
\text { muscle soreness } \\
\text { HR } \\
\text { RPE }\end{array}$ & 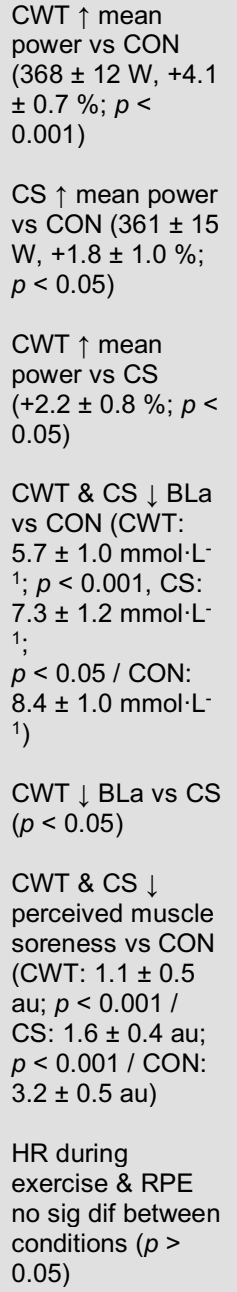 & 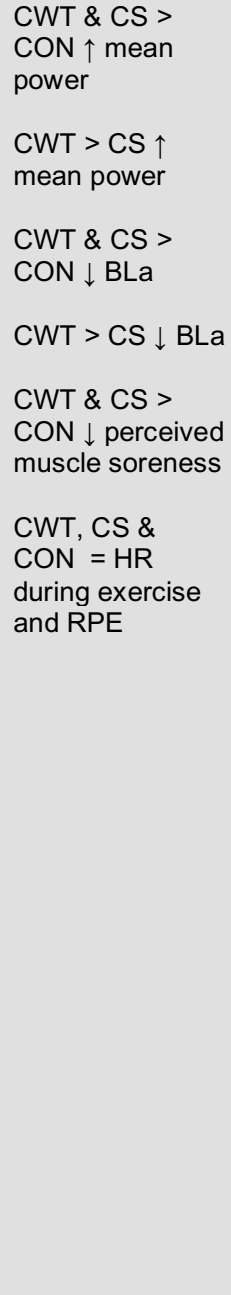 \\
\hline
\end{tabular}

$N$ number of cyclists, W/Kg watts per kilogram of bodyweight, COMP compression garment/full length tights, EMS electromyostimulation/electronic muscle stimulation, HUM humidification therapy, CON control condition/passive rest, BLa blood lactate concentration, TQR perceived total quality recovery, VO max maximal oxygen uptake, PPO peak power output, TT cycling time trial, CS compression stockings, rh relative humidity, HR heart rate, RPE ratings of perceived exertion, $C W T$ contrast water therapy.

colleagues (Degroot et al. 1993) have revealed that an increase in $\mathrm{Pi}$ and monovalent phosphate $\left(\mathrm{H}_{2} \mathrm{PO}_{4}^{-}\right)$, are better correlated with a reduction in maximum voluntary contraction than $\mathrm{H}^{+}$. An extensive review on the effects of metabolism end products and acidosis on muscle fatigue can be found elsewhere (Cairns 2006; Robergs et al. 2004; Westerblad et al. 2002). 


\section{Fatigue During Middle-Duration Cycling}

Middle-duration events in cycling range from a duration of between 2 to 30 -mins (Table 1). Therefore, the metabolic contribution from these events are highly dependent on the anaerobic glycolytic and aerobic system, with a minor contribution from the alactic system ( 1\%) (Jeukendrup et al. 2000). For example, in the male $4-\mathrm{km}$ TT ( $\sim 4 \mathrm{~min}$ duration) the anaerobic glycolytic system contributes $14 \%$ of energy production, while the aerobic system contributes a greater $85 \%$ of energy production (Jeukendrup et al. 2000). As a result of the high aerobic demand of cycling within this category, a limiting factor of performance is the ability of the cardiovascular system to supply sufficient oxygen to the working muscle (Abbiss and Laursen 2005). Middle-duration events occur on the severe intensity domain where power outputs are generated above critical power (CP) and sustained until $\mathrm{VO}_{2 \max }$ is achieved (Jones et al. 2010). Performing above CP during cycling tasks has been linked to a reduction of muscle PCr, ATP and a concomitant increase in $\mathrm{P}_{\mathrm{i}}$, plasma potassium ion $\left(\mathrm{K}^{+}\right)$and blood and muscle lactate (Black et al. 2017). A reduction in $\mathrm{PCr}$ and ATP concentration has been associated with an increase in electromyography (EMG) signals, demonstrating an attempt of the CNS to compensate for increased peripheral fatigue (Black et al. 2017). Moreover, a rise in extracellular $\mathrm{K}^{+}$will result in a decrease of action potential conduction, leading to a reduction of calcium ion $\left(\mathrm{Ca}^{2+}\right)$ release from the sarcoplasmic reticulum and a loss of contraction force (Allen et al. 2008).

\section{Fatigue During Endurance Cycling}

Endurance cycling events range from approximately 45 mins to 6-hrs (Table 1). Numerous models to explain fatigue during cycling within this category include but are not limited to; the energy depletion, metabolite accumulation, muscle trauma and neuromuscular fatigue models, and the reader is directed to an extensive review conducted elsewhere (Abbiss and Laursen 2005). Given the duration of these events, energy is predominantly produced from the aerobic system (Jeukendrup et al. 2000). As with middle-duration cycling, a limiting factor of performance is the ability of the cardiovascular system to supply sufficient oxygen to the working muscle (Abbiss and Laursen 2005). Furthermore, metabolic disturbances include a reduction in PCr, ATP, $\mathrm{pH}$ and glycogen, with a concomitant increase in blood and muscle lactate and $\mathrm{K}^{+}$; believed to disrupt $\mathrm{Ca}^{2+}$ release and result in a loss of contraction force (Black et al. 2017). Additionally, prolonged endurance cycling results in severe depletion of liver and muscle glycogen (Abbiss and Laursen 2005; Black et al. 2017) and reductions in voluntary strength (Millet and Lepers 2004). A further explanation for an increase in fatigue and consequent reduction in power output could be mechanical damage, resulting from muscle cell disruption (Mena et al. 1996).

\section{Recovery Modalities in Cycling}

Compression Garments (COMP)

Compression garments (COMP) are thought to improve exercise recovery through the application of pressure at the extremity i.e. ankle, thereby enhancing venous blood flow which in turn, assists in the removal of metabolic waste accumulated as a result of exercise (Argus et al. 2013). There are three types of COMP that have been examined in cycling literature: Compression stockings (Chatard et al. 2004; Ménétrier et al. 2013), full-length tights (Argus et al. 2013; Driller and Halson 2013) and dynamic compression (Overmayer and Driller, 2018). The ability of COMP to improve subsequent performance, perceived muscle soreness and muscle swelling, appears to be irrespective of garment type and pressure exerted, with both compression stockings and full-length tights, shown to attenuate the decrement in mean and maximal power, decrease thigh girth, calf girth and perceived muscle soreness post-recovery when compared with a passive control (CON) (Argus et al. 2013; Chatard et al. 2004; Driller and Halson 2013; Ménétrier et al. 2013). However, it is worth noting that not all studies quantified the actual pressure exerted by the garments used (Table 2). While Dynamic compression requires further studies, currently there seems to be no benefit when used between a 20-min and 4-min TT (Overmayer and Driller, 2018). COMP used for between $12-80$ mins post-exercise has been shown to improve 5-min maximal cycling mean and max power by up to $2.1 \%$, 30s cycling mean power by $0.8 \%$ (however the SD was $1.2 \%$ ) and 30-min cycling mean power by $2 \%$ (Table 3 ). Full length tights and compression stockings used for 12-80mins improved the rate of blood lactate $(\mathrm{BLa})$ removal following 10-min cycling beginning at $80 \%$ and increasing to $90 \%$ PPO, $30-$ min cycling beginning at $70 \%$ and increasing to $100 \%$ peak power output (PPO) and 5-mins of maximal cycling (Chatard et al. 2004; Driller and Halson 2013; Ménétrier et al. 2013). However, full length tights were no more beneficial than passive rest alone, at reducing BLa concentration following 30s of maximal sprint cycling (Argus et al. 2013). Furthermore, COMP resulted in no change in HR measures, TQR (Perceived Total Quality Recovery) or RPE (Rating of Perceived Exertion) when compared with CON (Argus et al. 2013; Chatard et al. 2004; Ménétrier et al. 2013). It should not be discounted that a placebo effect is responsible, at least in part, for the resultant performance benefits; a study by Argus and colleagues (Argus et al. 2013) attempted to account for a possible placebo effect through use of a belief questionnaire. Participants were required to predict whether or not the recovery intervention would enhance their recovery and results revealed that only $2 / 8$ participants accurately predicted the best strategy. Therefore, indicating that the placebo effect alone may not be responsible for the resultant performance benefits associated with COMP.

Future research should continue to use a valid and reliable method of pressure monitoring such as the Kikuhime (Brophy-Williams et al. 2014) to continue to examine whether there is a relationship between 
pressure exerted and resultant benefits in cyclists. To better understand whether a placebo effect is responsible for the benefits associated with COMP, researchers should continue to use a visual analogue scale (BrophyWilliams et al. 2016) to examine the placebo effect. Dynamic compression is a relatively new area of research and requires further examination in cyclists.

\section{Cold Water Immersion (CWI)}

Cold water immersion is the most researched recovery strategy in the cycling literature (Table 4). CWI has been suggested beneficial for the treatment of inflammation and perceived pain (Vaile et al. 2011). Due to the large number of studies examining CWI in cyclists, performance recovery and physiological variables will be examined separately for this recovery modality.

Three studies have reported improvements in power measures (Peiffer et al. 2008a; Stanley et al. 2013; Vaile et al. 2008b) while a further six studies report no significant difference following CWI (Buchheit et al. 2009; Chan et al. 2016; Christensen and Bangsbo 2016; Peiffer et al. 2008b; Stanley et al. 2012; Stanley et al. 2013) and only one study reported CWI as detrimental to power output (Schniepp et al. 2002). During a 4-km TT in the heat $\left(35^{\circ} \mathrm{C}\right)$, power output was reduced by 20 $\pm 6 \%$ in $\mathrm{CON}$, where CWI was able to attenuate this decrement to only a $3 \pm 3 \%$ reduction in power output and improved time to completion by $18 \pm 11.5 \mathrm{sec}$ (Peiffer et al. 2008a). During 66 ramped sprints beginning at $5 \mathrm{~s}$ and working up to $15 \mathrm{~s}$ per sprint, CWI was able to improve sprint power measures by $2.4 \%$ over 3 days (Stanley et al. 2013) and $1.4 \%$ over 5 days (Vaile et al. 2008b) when compared with CON. Following the aforementioned sprint cycling protocol, CWI improved 9-min TT mean power by up to $1 \%$ over 5 days, where $\mathrm{CON}$ reduced power by up to $3.8 \%$, this improvement in power was also associated with an improvement in total work performed on days $4 \& 5$ (Vaile et al. 2008b). In the studies exhibiting no improvement in power output from CWI, two studies utilised the same recovery protocol, which included 5mins of the condition and a further 15-mins passive seated (Buchheit et al. 2009; Peiffer et al. 2008b). Further studies had extensive recovery durations (over 2 hours) which may have diluted the impact of the recovery intervention (Christensen and Bangsbo 2016; Stanley et al. 2012). Stanley and colleagues (Stanley et al. 2013) reported no significant difference in power during TT cycling. However, these TT were preceded by 66 ramped sprints from which they saw CWI attenuated sprint power by up to $12 \%$ over 3 days when compared with CON; perhaps if the order of events were rotated in this study, an effect would have been observed. In the one study that revealed CWI was detrimental to performance (Schniepp et al. 2002), participants were required to push a very large gear, using a 53 tooth chainring and a 13 tooth rear sprocket, totalling 110 inches per cycle revolution in a short duration of $30 \mathrm{~s}$ and participants were confined to this one gear. This may have led to participants being unable to overcome the resistance effectively, while other participants could have found this resistance easier, especially considering there was a $9.9 \mathrm{~kg}$ deviation in weight and the level of experience varied among riders (category rank, training miles per year and races per year). Studies that examined subsequent performance and reported benefits from the use of CWI had an acclimation period consisting of a significant warm-up (Vaile et al. 2008b) or 10-mins passive rest post CWI (Peiffer et al. 2008a), where Schniepp and colleagues (Schniepp et al. 2002) required participants to towel dry and immediately remount their bicycles. Indeed, it has been suggested that a reduction in muscle temperature can impair cross-bridge cycling, motor unit activation and enzyme activity rate (Schniepp et al. 2002) which perhaps is mitigated by the use of passive rest or a warm-up post condition.

CWI $\left(15^{\circ} \mathrm{C}\right)$ used for 15 -mins and followed by 40 -mins passive rest, improved total worked performed; while AR ( $40 \%$ PPO) resulted in a reduction of total work performed (Vaile et al. 2011). In an earlier study by Vaile and colleagues (Vaile et al. 2008a) CWI was again superior when compared to AR and maintained 30-min cycling total work between bouts, while AR decreased total work by $4.1 \pm 1.8 \%$. Only one study revealed no significant difference in total work performed from the use of CWI (Peiffer et al. 2007) and can be attributed to a long recovery duration consisting of $1.5 \mathrm{hrs}$ before the performance trial (Peiffer et al. 2007).

CWI's impact on isometric and isokinetic force production following cycling is confounding. Maximum voluntary isometric contraction was reduced from the use of CWI $45 \& 90$-mins post $16.1 \mathrm{~km}$ TT when compared to CON (Peiffer et al. 2007). In this study, authors compared the use of electrical stimulation to examine if central inhibition was the limiting factor. However, as results revealed no significant difference between maximum voluntary isometric contraction and maximum voluntary isometric contraction with superimposed electrical stimulation, it was suggested that the limiting factor was related to a reduction in blood flow as examined by a reduction in venous vessel diameter 90 -mins post TT. Furthermore, later studies by the same author (Peiffer et al. 2008b; Peiffer et al. 2009) revealed no significant difference in isometric and isokinetic torque.

CWI decreased HR post-recovery by $4.2 \%$ when used for 15-mins between sprint cycling of 30s (Schniepp et al. 2002). In addition to improved HR post-recovery, CWI consistently increased HRV measures with large effect sizes (Buchheit et al. 2009; Stanley et al. 2012; Stanley et al. 2013).

Perceived recovery measures revealed that CWI improved ratings of perceived physical and mental recovery, reduced perceived muscle soreness and perceived general fatigue (Buchheit et al. 2009; Halson et al. 2008). Stanley and colleagues (Stanley et al. 2012; Stanley et al. 2013) also revealed similar improvements in a reduction of perceived general fatigue, leg soreness 
and an increase in physical recovery however, no significant difference and unclear effect sizes were observed in mental recovery and perceived tiredness. Christensen and Bangsbo (2016) was the only study to examine perceived readiness and results revealed there was no change between conditions.

BLa results revealed no significant difference following a 4-min TT (Christensen and Bangsbo 2016) and a 40min TT in heat (Halson et al. 2008). Unfortunately subsequent performance wasn't examined in these studies.

Table 4. Summary of studies examining the use of cold water immersion post-exercise in cyclists.

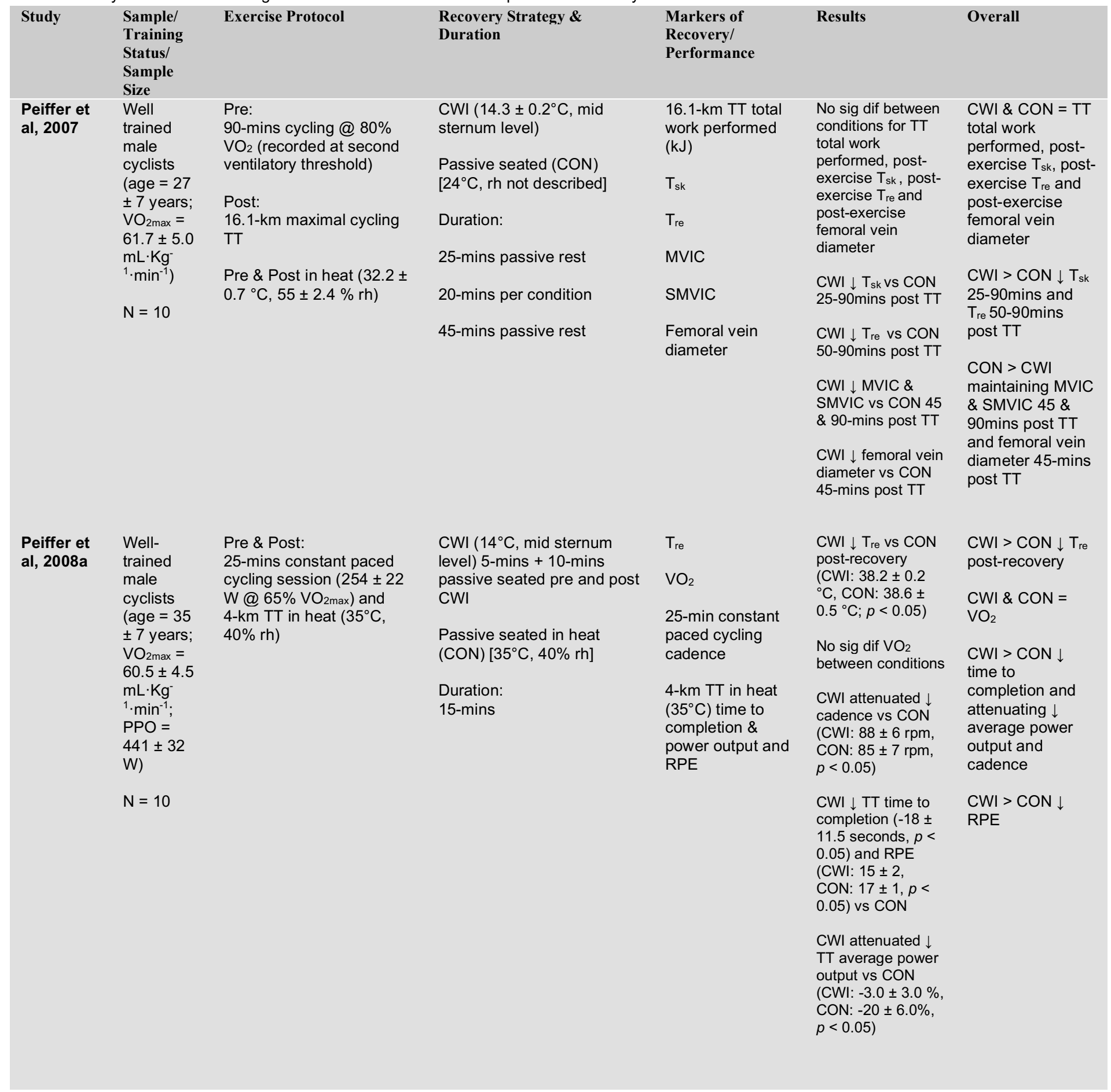




\begin{tabular}{|c|c|c|c|c|c|c|}
\hline $\begin{array}{l}\text { Peiffer et } \\
\text { al, 2008b }\end{array}$ & $\begin{array}{l}\text { Male } \\
\text { cyclists } \\
(\text { age }=29 \pm \\
6 \text { years; } \\
\mathrm{VO}_{2 \mathrm{max}}= \\
56.5 \pm 5.0 \\
\mathrm{~mL} \cdot \mathrm{Kg}^{-} \\
\left.{ }^{1} \cdot \mathrm{min}^{-1}\right) \\
\mathrm{N}=10\end{array}$ & $\begin{array}{l}\text { Pre \& post: } \\
1 \text {-km cycling TT in heat } \\
\left(35 \pm 0.3^{\circ} \mathrm{C}, 40 \pm 3 \% \mathrm{rh}\right)\end{array}$ & $\begin{array}{l}\mathrm{CWI}\left(14^{\circ} \mathrm{C} \text {, mid sternal }\right. \\
\text { level) } 5 \text {-mins }+15 \text {-mins } \\
\text { passive seated } \\
20 \text {-mins passive seated } \\
(\mathrm{CON})\left[35^{\circ} \mathrm{C}, 40 \% \mathrm{rh}\right] \\
\text { Duration: } \\
20 \text {-mins }\end{array}$ & $\begin{array}{l}T_{\text {re }} \\
\text { Isokinetic torque } \\
T_{\text {mus }} \\
\text { PPO } \\
\text { Mean power } \\
\text { Time to } \\
\text { completion }\end{array}$ & $\begin{array}{l}\text { Tre and isokinetic } \\
\text { quadriceps torque } \\
\text { no sig dif post- } \\
\text { recovery between } \\
\text { conditions } \\
\\
\text { CWI } \downarrow \text { quadriceps } \\
\mathrm{T}_{\text {mus }} \text { (CWI: } 36.4 \pm \\
0.8^{\circ} \mathrm{C}, \mathrm{CON}: 37.7 \\
\pm 0.3^{\circ} \mathrm{C}, p< \\
0.001 \text { ) } \\
\text { No sig dif PPO, } \\
\text { average power } \\
\text { and time to } \\
\text { completion } \\
\text { between } \\
\text { conditions }(p= \\
0.42 \text { to } 0.50)\end{array}$ & $\begin{array}{l}\mathrm{CWI}>\mathrm{CON} \downarrow \\
\text { quadriceps Tmus } \\
\text { in heat } \\
\mathrm{CWI} \& \mathrm{CON}= \\
\mathrm{PPO} \text {, average } \\
\text { power, time to } \\
\text { completion and } \\
\text { rectal } \\
\text { temperature in } \\
\text { heat }\end{array}$ \\
\hline $\begin{array}{l}\text { Peiffer et } \\
\text { al, } 2009\end{array}$ & $\begin{array}{l}\text { Male } \\
\text { cyclists } \\
\text { (age }=29 \pm \\
3 \text { years; } \\
\mathrm{VO}_{2 \max }= \\
64.0 \pm 5.7 \\
\mathrm{~mL} \cdot \mathrm{Kg}^{-} \\
1 \cdot \mathrm{min}^{-1} ; \\
\mathrm{PPO}=435 \\
\pm 45 \mathrm{~W}) \\
\mathrm{N}=12\end{array}$ & $\begin{array}{l}\text { Pre: } \\
\text { Cycling time to } \\
\text { exhaustion test in heat } \\
\left(40^{\circ} \mathrm{C}, 40 \% \text { rh, } 57 \pm 7 \%\right. \\
\left.\mathrm{VO}_{2 \max }\right)\end{array}$ & $\begin{array}{l}\mathrm{CWI} \times 5 \text {-mins }(\mathrm{CWI5}) \\
{\left[14^{\circ} \mathrm{C}, \text { mid sternum level] }\right.} \\
\mathrm{CWI} \times 10 \text {-mins }(\mathrm{CWI} 10) \\
{\left[14^{\circ} \mathrm{C}, \text { mid sternum level] }\right.} \\
\mathrm{CWI} \times 20 \text {-mins }(\mathrm{CWI} 20) \\
{\left[14^{\circ} \mathrm{C}, \text { mid sternum level] }\right.} \\
\text { Passive seated } \times 20 \text {-mins } \\
(\mathrm{CON})\left[24^{\circ} \mathrm{C}\right] \\
\text { Duration: } \\
25-m i n s \text { passive seated } \\
\left(24^{\circ} \mathrm{C}, \text { rh not described }\right) \\
\text { Condition duration above }\end{array}$ & $\begin{array}{l}\text { Time to } \\
\text { exhaustion (min) } \\
\text { Total work } \\
\text { performed (kJ) } \\
T_{\text {re }} \\
T_{\text {mus }} \\
\text { Isometric and } \\
\text { isokinetic torque }\end{array}$ & 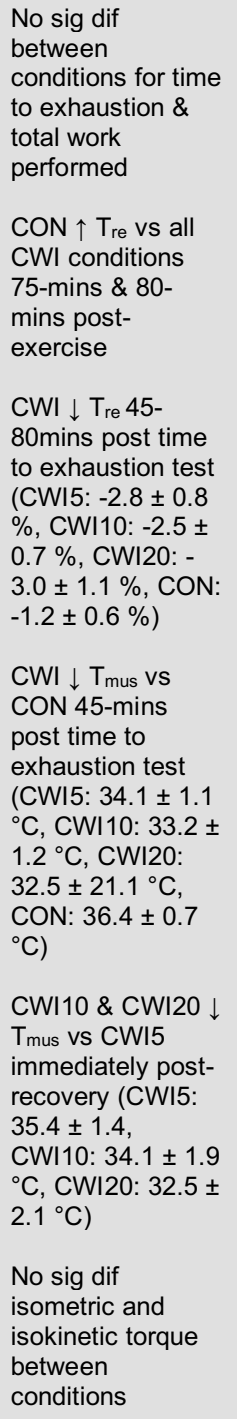 & $\begin{array}{l}\text { CWI5, CWI10, } \\
\text { CWI20 \& CON = } \\
\text { time to } \\
\text { exhaustion and } \\
\text { total work } \\
\text { performed } \\
\text { CON > CWI5, } \\
\text { CWI10 \& CWI20 } \\
\uparrow T_{\text {re }} 75 \text { \& 80- } \\
\text { mins post } \\
\text { exercise } \\
\text { CWI5, CWI10 \& } \\
\text { CWI20 > CON } \downarrow \\
T_{\text {re } 45-80 \text { mins }} \\
\text { post time to } \\
\text { exhaustion test } \\
\text { CWI5, CWI10 \& } \\
\text { CWI20 > CON } \downarrow \\
\text { muscle } \\
\text { temperature } 45- \\
\text { mins post time to } \\
\text { exhaustion test } \\
\text { CWI10 \& CWI20 } \\
>\text { CWI5 } \downarrow \text { muscle } \\
\text { temperature } \\
\text { immediately } \\
\text { post-recovery } \\
\text { CWI5, CWI10, } \\
\text { CWI20 \& CON = } \\
\text { isometric and } \\
\text { isokinetic torque }\end{array}$ \\
\hline
\end{tabular}




\begin{tabular}{|c|c|c|c|c|c|c|}
\hline $\begin{array}{l}\text { Halson et } \\
\text { al, } 2008\end{array}$ & $\begin{array}{l}\text { Male } \\
\text { endurance } \\
\text { trained } \\
\text { cyclists (age } \\
=23.8 \pm 1.6 \\
\text { years; } \\
\mathrm{VO}_{2 \max }= \\
71.3 \pm 1.2 \\
\mathrm{~mL} \cdot \mathrm{Kg}^{-} \\
{ }^{1} \cdot \mathrm{min}^{-1} \text { ) } \\
\mathrm{N}=11\end{array}$ & $\begin{array}{l}\text { Pre: } \\
\sim 40-\text { min TT in heat ( } 34.3 \pm \\
\left.1.1^{\circ} \mathrm{C}, 41.2 \pm 3.0 \% \mathrm{rh}\right)- \\
\text { first } 20 \text {-mins fixed } \\
\text { workload, final } 20 \text {-mins } \\
\text { same amount of work (kJ) } \\
\text { as first } 20 \text {-min but } \\
\text { completed as quickly as } \\
\text { possible }\end{array}$ & $\begin{array}{l}\mathrm{CWI}\left(11.5^{\circ} \mathrm{C} \text {, mesosternal }\right. \\
\text { height) } \\
\text { Passive recovery }(\mathrm{CON}) \\
{\left[24.2 \pm 1.8^{\circ} \mathrm{C}, 45.6 \pm 6.5 \%\right.} \\
\text { rh] } \\
\text { Duration: } \\
20 \text {-mins passive rest } \\
\text { followed by } \\
3 \times 60 \text { s per conditions with } \\
2 \text {-mins seated rest between } \\
{\left[24.2 \pm 1.8^{\circ} \mathrm{C}, 45.6 \pm 6.5 \%\right.} \\
\text { rh] }\end{array}$ & $\begin{array}{l}\text { HR } \\
\mathrm{T}_{\text {re }} \\
\text { BLa } \\
\mathrm{T}_{\text {sk }} \\
\text { Mean body } \\
\text { temperature } \\
\text { Cooling rate } \\
\text { pH, chloride, } \\
\text { glucose, } \\
\text { bicarbonate, } \\
\text { potassium, } \\
\text { sodium, PCO } \\
\text { PO, } \\
\text { IGF-1, } \\
\text { testosterone, GH, } \\
\text { plasma CRP, IL- } \\
6, \text { cortisol } \\
\text { concentration, } \\
\text { plasma prolactin } \\
\text { concentration, } \\
\text { plasma } \\
\text { adrenaline, } \\
\text { plasma } \\
\text { noradrenaline } \\
\text { Ratings of } \\
\text { perceived: } \\
\text { Physical, mental, } \\
\text { muscular } \\
\text { recovery and } \\
\text { general fatigue }\end{array}$ & 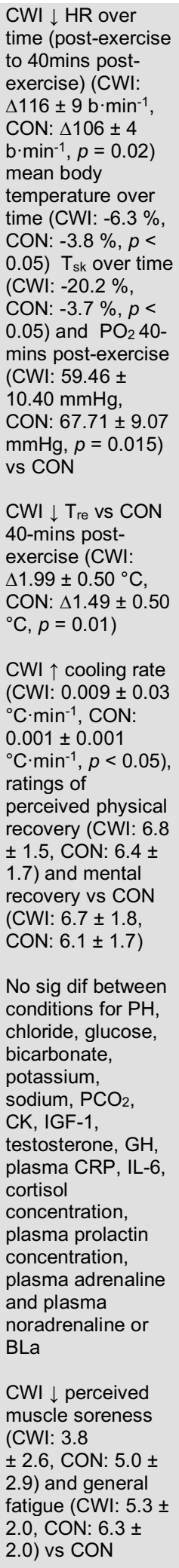 & $\begin{array}{l}\mathrm{CWI}>\mathrm{CON} \downarrow \\
\mathrm{HR}, \mathrm{T}_{\text {re, }} \mathrm{T}_{\text {sk }} \text { and } \\
\text { mean body } \\
\text { temperature } \\
\mathrm{CWI} \& \mathrm{CON}= \\
\text { BLa, PH, } \\
\text { chloride, glucose, } \\
\text { bicarbonate, } \\
\text { potassium, } \\
\text { sodium, PCO }{ }_{2} \text {, } \\
\text { CK, IGF-1, } \\
\text { testosterone, GH, } \\
\text { plasma CRP, IL- } \\
6, \text { cortisol } \\
\text { concentration, } \\
\text { plasma prolactin } \\
\text { concentration, } \\
\text { plasma } \\
\text { adrenaline and } \\
\text { plasma } \\
\text { noradrenaline } \\
\text { CWI > CON } \uparrow \\
\text { cooling rate } \\
\text { CWI > CON } \downarrow \\
\text { PO }{ }_{2} 40-\text { mins } \\
\text { post-exercise } \\
\text { CWI > CON } \uparrow \\
\text { perceived } \\
\text { physical recovery } \\
\text { and mental } \\
\text { recovery } \\
\mathrm{CWI} \mathrm{>} \mathrm{CON} \downarrow \\
\text { perceived muscle } \\
\text { soreness and } \\
\text { general fatigue }\end{array}$ \\
\hline
\end{tabular}




\begin{tabular}{|c|c|c|c|c|c|c|}
\hline $\begin{array}{l}\text { Stanley et } \\
\text { al, } 2012\end{array}$ & $\begin{array}{l}\text { Endurance } \\
\text { trained } \\
\text { male } \\
\text { cyclists } \\
\text { (age }=27 \pm \\
7 \text { years; } \\
\mathrm{VO}_{2 \max }= \\
63.9 \pm 7.2 \\
\mathrm{~mL} \cdot \mathrm{Kg}^{-} \\
{ }^{1} \cdot \mathrm{min}^{-1} ; \\
\mathrm{PPO}=418 \\
\pm 40 \mathrm{~W} \text { ) } \\
\mathrm{N}=18\end{array}$ & $\begin{array}{l}\text { Pre: } \\
8 \times 4 \text {-mins cycling @ 80\% } \\
\text { PPO with 1-min AR (50\% } \\
\text { PPO) between intervals } \\
\text { Post: } \\
\text { Performance trial } \\
\text { (standardized amount of } \\
\text { work = 75\% PPO x 15- } \\
\text { mins) }\end{array}$ & $\begin{array}{l}\mathrm{CWI}\left(14 \pm 1^{\circ} \mathrm{C} \text {, shoulder }\right. \\
\text { height) } \\
\mathrm{CWT}(1 \text {-min } \mathrm{CWI}[14 \pm \\
\left.1^{\circ} \mathrm{C}\right], 3 \times 2 \text {-mins HWI }[40 \pm \\
\left.1^{\circ} \mathrm{C}\right] \text { and ending with } 1 \text {-min } \\
\mathrm{CWI}) \\
\text { Passive rest (CON) }\left[22^{\circ} \mathrm{C} \text {, }\right. \\
\text { rh not described] } \\
\text { Duration: } \\
20 \text {-mins post-exercise } \\
\text { each conditions } \\
\text { implemented: } \\
\text { CWI = 5-mins + 5-mins } \\
\text { passive seated } \\
\text { CWT = 10-mins } \\
\text { CON = 10-mins } \\
\text { An additional } 160 \text {-mins } \\
\text { passive seated for all } \\
\text { conditions }\end{array}$ & $\begin{array}{l}\text { Time to } \\
\text { completion } \\
\text { HR } \\
\text { HR }_{\text {max }} \\
\text { Power output } \\
\Delta \text { rMSSD } \\
\text { (baseline vs } \\
\text { during passive } \\
\text { recovery) } \\
\text { Perceived: } \\
\text { General fatigue, } \\
\text { mental recovery, } \\
\text { leg soreness, } \\
\text { physical } \\
\text { recovery }\end{array}$ & $\begin{array}{l}\text { No sig dif } \\
\text { between } \\
\text { conditions for HR } \\
\text { and HRmax (during } \\
\text { performance trial), } \\
\text { time to } \\
\text { completion, power } \\
\text { output and } \\
\text { perceived mental } \\
\text { recovery } \\
\text { CWI } \downarrow \text { HR during } \\
\text { first } 10 \% \text { of } \\
\text { performance trial } \\
\text { vs CON \& CWT } \\
\text { (likely lower) } \\
\text { CWI } \downarrow \text { power } \\
\text { output during first } \\
10 \% \text { of } \\
\text { performance trial } \\
\text { vs CON (likely } \\
\text { lower) } \\
\text { CWT } \uparrow \text { power } \\
\text { output between } \\
40-80 \% \text { the } \\
\text { duration of the } \\
\text { performance trial } \\
\text { vs CON (very } \\
\text { likely higher) } \\
\text { CWI \& CWT } \uparrow \\
\text { ArMSSD vs CON } \\
\text { (large effect size) } \\
\text { CWI } \uparrow \text { rMSSD vs } \\
\text { CWT (small effect } \\
\text { size) } \\
\text { CWI } \downarrow \text { perceived } \\
\text { general fatigue vs } \\
\text { CON (very likely } \\
\text { lower) } \\
\text { CWT } \downarrow \text { perceived } \\
\text { general fatigue vs } \\
\text { CON (likely lower) } \\
\text { CWI \& CWT } \downarrow \\
\text { perceived leg } \\
\text { soreness vs CON } \\
\text { (almost certainly } \\
\text { lower) } \\
\text { CWI } \uparrow \text { perceived } \\
\text { physical recovery } \\
\text { vs CON (possibly } \\
\text { higher) } \\
\text { CWT } \uparrow \text { perceived } \\
\text { physical recovery } \\
\text { vs CON (likely } \\
\text { higher) }\end{array}$ & $\begin{array}{l}\text { CWI, CWT \& } \\
\text { CON = HR, } \\
\text { HRmax, time to } \\
\text { completion, } \\
\text { power output } \\
\text { and perceived } \\
\text { mental recovery } \\
\text { CON }>\text { CWI } \\
\text { maintaining HR } \\
\text { and power } \\
\text { output during } \\
\text { first } 10 \% \text { of } \\
\text { performance trial } \\
\text { duration } \\
\text { CWT > CON } \uparrow \\
\text { power output } \\
\text { between } 40-80 \% \\
\text { duration of } \\
\text { performance trial } \\
\text { CWI \& CWT > } \\
\text { CON } \uparrow \Delta \mathrm{rMSSD} \\
\text { and } \downarrow \text { perceived } \\
\text { leg soreness } \\
\text { CWI }>\text { CWT } \uparrow \\
\Delta \mathrm{rMSSD} \downarrow \text { and } \\
\text { perceived } \\
\text { general fatigue } \\
\text { CWI }>\text { CON } \downarrow \\
\text { perceived } \\
\text { general fatigue } \\
\text { and } \uparrow \text { perceived } \\
\text { physical } \\
\text { recovery } \\
\text { CWT > CWI } \uparrow \\
\text { perceived } \\
\text { physical } \\
\text { recovery }\end{array}$ \\
\hline
\end{tabular}




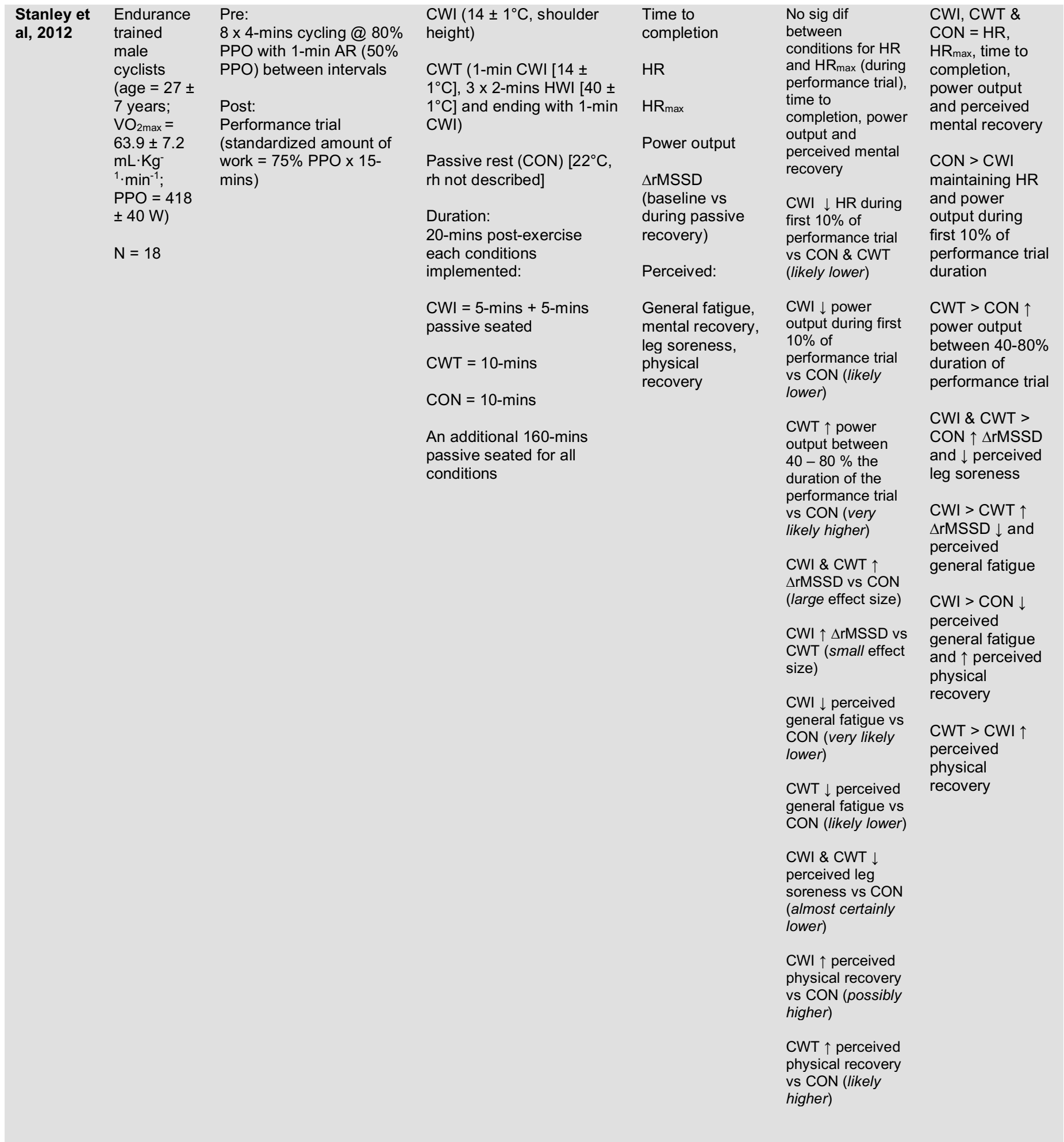




\begin{tabular}{|c|c|c|c|c|c|c|}
\hline $\begin{array}{l}\text { Vaile } \\
\text { et al, } \\
2008 a\end{array}$ & $\begin{array}{l}\text { Well- } \\
\text { trained } \\
\text { male } \\
\text { cyclists } \\
\text { (age }=32 \pm \\
5 \text { years; } \\
\mathrm{VO}_{2 \max }= \\
70.7 \pm 7.9 \\
\mathrm{~mL} \cdot \mathrm{Kg}^{-} \\
{ }^{1} \cdot \mathrm{min}^{-1} \text { ) } \\
\mathrm{N}=10\end{array}$ & $\begin{array}{l}\text { Pre (Ex1): } \\
30-\text {-min cycling in heat }(34 \\
\pm 0.2^{\circ} \mathrm{C}, 39.4 \pm 1.5 \% \text { rh, } \\
15 \text {-min @ } 70 \% \text { PPO and } \\
\text { a } 15 \text {-min maximal cycling } \\
\text { TT) } \\
\text { Post (Ex } 2) \text { : } \\
30 \text {-min cycling in heat ( } 34 \\
\pm 0.2^{\circ} \mathrm{C}, 39.4 \pm 1.5 \% \text { rh, } \\
15 \text {-min @ } 70 \% \text { PPO and } \\
\text { a } 15-\text { min maximal cycling } \\
\text { TT) }\end{array}$ & $\begin{array}{l}\text { Shoulder height for all CWI } \\
\text { conditions } \\
\text { Intermittent CWI, } 10^{\circ} \mathrm{C} \\
\text { (ICWI10) } \\
\text { Intermittent CWI, } 15^{\circ} \mathrm{C} \\
\text { (ICWI15) } \\
\text { Intermittent CWI, } 20^{\circ} \mathrm{C} \\
\text { (ICWI20) } \\
\text { Continuous CWI, } 20^{\circ} \mathrm{C} \text {, in } \\
\text { bath for entire } 15-\text {-mins } \\
\text { (CCWI20) } \\
\text { AR (15-mins @ } 40 \% \\
\left.\text { VO2max, } 31.1 \pm 2.6^{\circ} \mathrm{C}\right) \\
\text { Duration: } \\
\text { Intermittent CWI }=5 \times 1 \text { - } \\
\text { min in bath, } 2 \text {-mins out of } \\
\text { bath }\left(29.2 \pm 1.4{ }^{\circ} \mathrm{C}, 58 \pm\right. \\
2.1 \% \text { rh) } \\
15 \text {-mins total per condition } \\
40-\text { mins passive recovery } \\
\left(34 \pm 0.2^{\circ} \mathrm{C}, 39.4 \pm 1.5 \%\right. \\
\text { rh) }\end{array}$ & $\begin{array}{l}\text { 30-min cycling } \\
\text { total work } \\
(\mathrm{kJ}) \\
\text { Body } \\
\text { temperature } \\
\text { BLa } \\
\text { RPE } \\
\text { HR }_{\text {post-intervention }} \\
\text { HR }_{\text {post-recovery }}\end{array}$ & 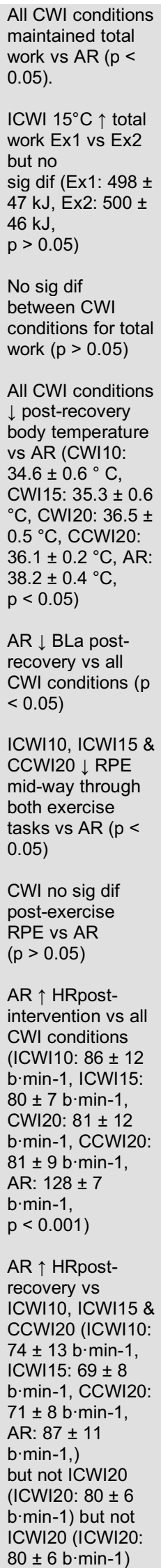 & $\begin{array}{l}\text { All CWI } \\
\text { conditions > AR } \\
\text { maintaining total } \\
\text { work and } \downarrow \text { post- } \\
\text { recovery body } \\
\text { temperature } \\
\text { AR > all CWI } \\
\text { conditions } \downarrow \text { BLa } \\
\text { ICWI10, ICWI15, } \\
\text { CCWI20 > AR } \downarrow \\
\text { RPE during } \\
\text { exercise } \\
\text { All CWI } \\
\text { conditions \& AR } \\
=\text { RPE post- } \\
\text { exercise } \\
\text { AR }>\text { all CWI } \\
\text { conditions } \uparrow \\
\text { HR post-intervention } \\
\text { AR > ICWI10, } \\
\text { ICWI15 \& } \\
\text { CCWI20 } \uparrow \\
\text { HR }\end{array}$ \\
\hline
\end{tabular}




\begin{tabular}{|c|c|c|c|c|c|c|}
\hline $\begin{array}{l}\text { Vaile } \\
\text { et al, } \\
2008 b\end{array}$ & $\begin{array}{l}\text { Endurance } \\
\text { trained } \\
\text { male } \\
\text { cyclists } \\
\text { (age }=32.2 \\
\pm 4.3 \text { years; } \\
\mathrm{VO}_{2 \mathrm{max}}= \\
68.8 \pm 3.6 \\
\mathrm{~mL} \cdot \mathrm{Kg}^{-} \\
{ }^{1} \cdot \mathrm{min}^{-1} \text { ) } \\
\mathrm{N}=12\end{array}$ & $\begin{array}{l}\text { Pre: } \\
5 \text { consecutive days - } \\
66 \text { max sprints }(5-15 \mathrm{~s} \\
\text { with a specific work to } \\
\text { rest ratio of } 1: 6,1: 3 \text { or } 1: 1 \\
\text { - rest is AR @ } 40-50 \% \\
\text { PPO) + 9-min TT }(2 \times 2- \\
\text { min \& } 1 \times 5 \text {-min) }\end{array}$ & $\begin{array}{l}\mathrm{CWI}\left(15^{\circ} \mathrm{C} \text {, shoulder }\right. \\
\text { height) } \\
\text { HWI }\left(38^{\circ} \mathrm{C} \text {, shoulder }\right. \\
\text { height) } \\
\mathrm{CWT}\left(7 \times 15^{\circ} \mathrm{C} 1 \text {-min; }\right. \\
38^{\circ} \mathrm{C} 1 \text {-min, shoulder } \\
\text { height) } \\
\text { Passive seated (CON) } \\
\text { [room temperature and } \\
\text { humidity not stipulated] } \\
\text { Duration: } \\
\text { 14-mins }\end{array}$ & $\begin{array}{l}\text { Sprints: } \\
\text { Mean power } \\
\text { TT: } \\
\text { TT total work } \\
\text { performed (kJ) } \\
\text { Mean power } \\
\text { Tre } \\
\text { HR } \\
\text { RPE }\end{array}$ & 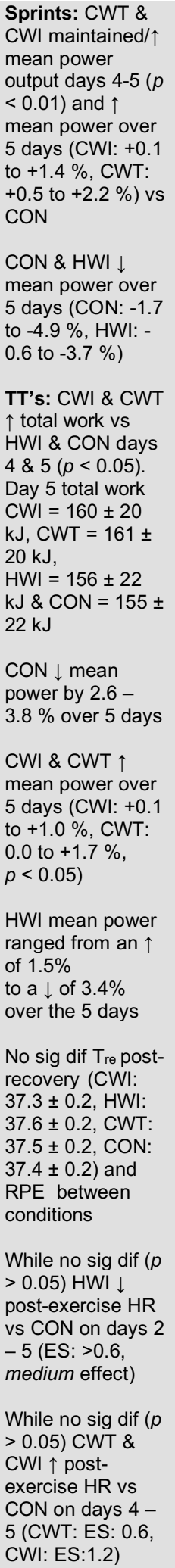 & $\begin{array}{l}\text { CWT \& CWI > } \\
\text { CON } \\
\text { maintaining/ } \uparrow \\
\text { sprint mean } \\
\text { power output } \\
\text { days 4-5 } \\
\text { CWT \& CWI > } \\
\text { HWI \& CON } \uparrow \\
\text { TT total work } \\
\text { performed } \\
\text { CWT \& CWI > } \\
\text { HWI \& CON } \uparrow \\
\text { TT mean power } \\
\text { output over } 5 \\
\text { days } \\
\text { CWT, CWI, HWI } \\
\& \text { CON = Tre } \\
\text { post-recovery } \\
\text { HWI > CWT, } \\
\text { CWI \& CON } \downarrow \\
\text { HR post- } \\
\text { exercise days } 2- \\
5\end{array}$ \\
\hline
\end{tabular}




\begin{tabular}{|c|c|c|c|c|c|c|}
\hline $\begin{array}{l}\text { Vaile } \\
\text { et al, } 2011\end{array}$ & $\begin{array}{l}\text { Endurance } \\
\text { trained } \\
\text { male } \\
\text { cyclists } \\
\text { (age }=33.7 \\
\pm 4.7 \text { years; } \\
\mathrm{VO}_{2 \max }= \\
66.7 \pm 6.1 \\
\mathrm{~mL} \cdot \mathrm{Kg}^{-} \\
{ }^{1} \cdot \mathrm{min}^{-1} \\
\mathrm{~N}=10\end{array}$ & $\begin{array}{l}\text { Pre \& post: } \\
35 \text {-mins cycling in heat } \\
{\left[32.8 \pm 1.1^{\circ} \mathrm{C}, 43.6 \pm 1.8\right.} \\
\% \text { rh] }(15-\text {-mins @ } 70 \% \\
\text { PPO; } 15 \text {-min TT) }\end{array}$ & $\begin{array}{l}\mathrm{CWI}\left(15^{\circ} \mathrm{C} \text {, shoulder }\right. \\
\text { height) } \\
\text { AR @ } 40 \% \text { PPO }(32.8 \pm \\
\left.1.1^{\circ} \mathrm{C}\right) \\
\text { Duration: } \\
15 \text {-mins per conditions } \\
\text { Passive rest in a supine } \\
\text { position for } 40 \text {-mins }(32.8 \pm \\
\left.1.1^{\circ} \mathrm{C}, 43.6 \pm 1.8 \% \mathrm{rh}\right)\end{array}$ & $\begin{array}{l}\text { 15-min TT total } \\
\text { work performed } \\
\text { (kJ) } \\
T_{\text {re }} \\
\text { Limb blood flow } \\
\text { (arm blood flow, } \\
\text { leg blood flow \& } \\
\text { leg to arm blood } \\
\text { flow ratio) } \\
\text { HR } \\
\text { BLa }\end{array}$ & $\begin{array}{l}\text { AR } \downarrow \text { total work } \\
\text { performed (pre to } \\
\text { post } \Delta:-1.8 \pm-1.1 \\
\%) \\
\text { CWI } \uparrow \text { total work } \\
\text { performed } \\
\text { (pre to post } \Delta \text { : } \\
+0.10 \pm 0.7 \% \text { ) } \\
\text { CWI } \downarrow \text { Tre post- } \\
\text { recovery and } \\
\text { post-exercise } \\
(p<0.05) \\
\text { CWI } \downarrow \text { leg and } \\
\text { arm blood flow vs } \\
\text { AR during } \\
\text { recovery and } \\
\text { post-recovery } \\
\text { CWI } \downarrow \text { arm blood } \\
\text { flow post-exercise } \\
\text { vs AR } \\
\text { ( } p<0.05 \text { ) } \\
\text { CWI } \uparrow \text { leg to arm } \\
\text { blood flow ratio vs } \\
\text { AR during } \\
\text { recovery } \\
\text { No sig dif post- } \\
\text { exercise blood } \\
\text { flow ratio between } \\
\text { conditions } \\
\text { CWI } \downarrow \text { HR during } \\
\text { and post recovery } \\
\text { vs AR (CWl: } 78 \pm \\
15 \text { b min }{ }^{-1}, \text { AR: } 90 \\
\pm 11 \text { b minn }-1 \\
p<0.05 \text { ) } \\
\text { CWI } \downarrow \text { HR during } \\
\text { first } 5 \text {-mins of } \\
\text { exercise vs AR } \\
\text { AR } \downarrow \text { BLa post- } \\
\text { recovery vs CWI } \\
\text { (CWl: } 4.5 \pm 1.2 \\
\text { mM, AR: } 2.3 \pm 0.8 \\
\text { mM, } p<0.05 \text { ) }\end{array}$ & $\begin{array}{l}\mathrm{CWI}>\mathrm{AR} \uparrow \text { total } \\
\text { work performed } \\
\mathrm{CWI}>\mathrm{AR} \downarrow \mathrm{T} \text { re } \\
\text { leg and arm } \\
\text { blood flow during } \\
\text { recovery } \\
\mathrm{CWI}>\mathrm{AR} \uparrow \text { leg } \\
\text { to arm blood flow } \\
\text { ratio during } \\
\text { recovery } \\
\mathrm{CWI} \& \mathrm{AR}=\text { leg } \\
\text { to blood flow } \\
\text { ratio post- } \\
\text { exercise } \\
\mathrm{CWI}>\mathrm{AR} \downarrow \mathrm{HR} \\
\mathrm{AR}>\mathrm{CWI} \downarrow \mathrm{BLa}\end{array}$ \\
\hline
\end{tabular}




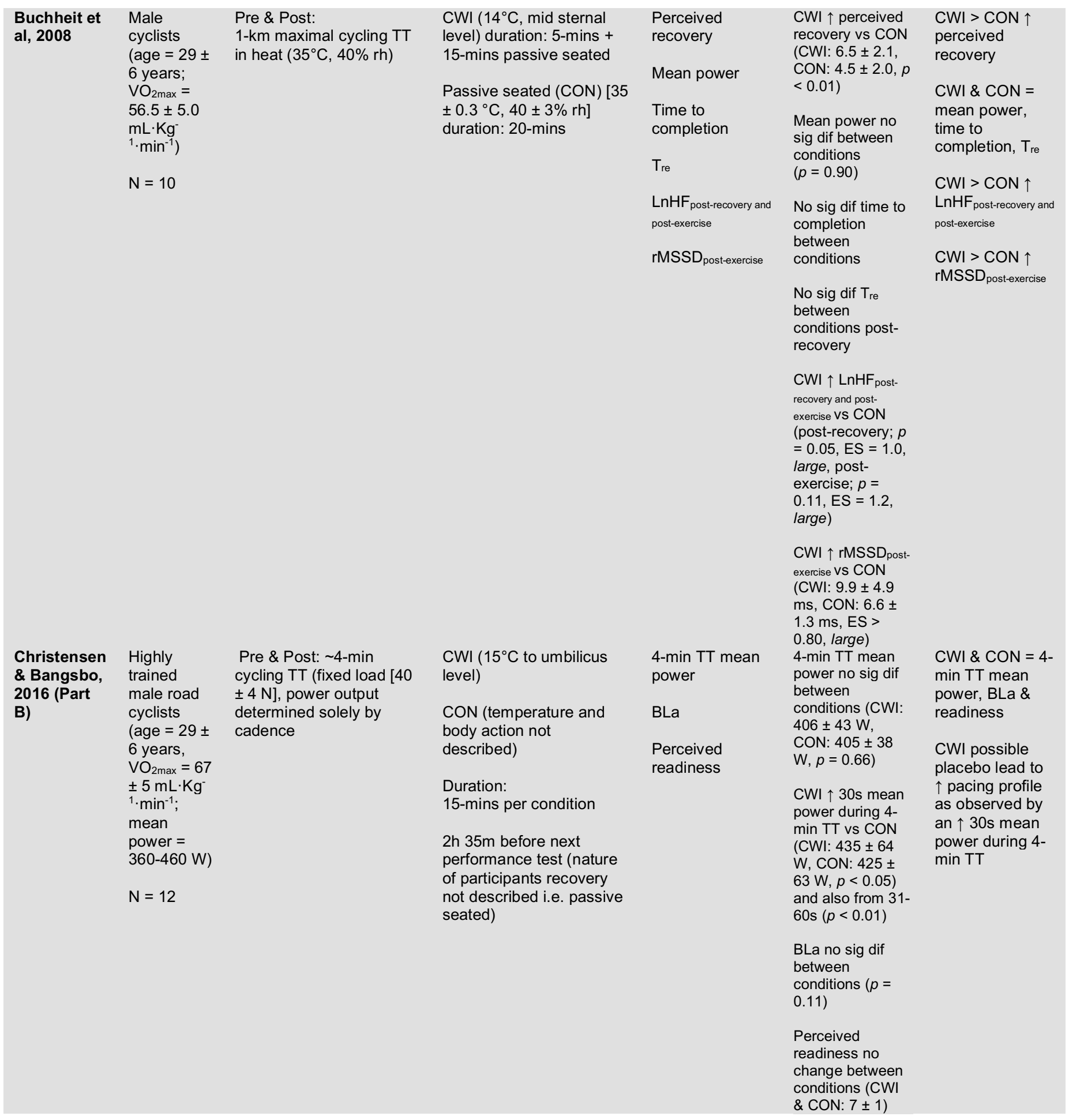




\begin{tabular}{|c|c|c|c|c|c|c|}
\hline $\begin{array}{l}\text { Chan et } \\
\text { al, } 2016\end{array}$ & $\begin{array}{l}\text { Junior elite } \\
\text { male } \\
\text { cyclists } \\
\text { (age }=16 \pm \\
1 \text { year; } \\
\mathrm{VO}_{2 \max }= \\
64.7 \pm 4.3 \\
\mathrm{~mL} \cdot \mathrm{Kg}^{-} \\
{ }^{1} \cdot \mathrm{min}^{-1} \\
\mathrm{~N}=8\end{array}$ & $\begin{array}{l}\text { Pre: } \\
\text { 15-mins cycling @ } 75 \% \\
\text { PPO \& 15-min TT in heat } \\
\text { (TT1, 31 }{ }^{\circ} \mathrm{C}, 74 \% \text { rh) } \\
\text { Post: } \\
15 \text {-mins cycling @ } 75 \% \\
\text { PPO \& 15-min TT in heat } \\
\text { (TT2, } 31^{\circ} \mathrm{C}, 74 \% \text { rh) }\end{array}$ & $\begin{array}{l}\mathrm{CWI}\left(15^{\circ} \mathrm{C} \text {, mid-sternum }\right. \\
\text { level) } \\
\text { CCT }\left(15^{\circ} \mathrm{C} \text {, ankle and }\right. \\
\text { thigh of both legs, rhythmic } \\
\text { compression setting HIGH) } \\
\text { AR @ } 40 \% \text { PPO }\left(31^{\circ} \mathrm{C}\right) \\
\text { Duration: } \\
10 \text {-mins passive seated in } \\
\text { heat }\left(31^{\circ} \mathrm{C}, 74 \% \text { rh }\right) \text {, } \\
15 \text {-mins per condition, } \\
30 \text {-mins passive seated in } \\
\text { heat }\end{array}$ & $\begin{array}{l}\text { Mean power } \\
\text { Core body } \\
\text { temperature } \\
\text { BLa } \\
\text { RPE } \\
\text { HR }_{\text {recovery }}\end{array}$ & $\begin{array}{l}\text { No sig dif TT2 } \\
\text { mean power } \\
\text { between } \\
\text { conditions ( } p= \\
0.551 \text { ) } \\
\text { CWI } \downarrow \text { core body } \\
\text { temperature 15- } \\
\text { mins } \\
\text { during recovery vs } \\
\text { CCT ( } p=0.011 \text { ) } \\
\text { CWI } \downarrow \text { core body } \\
\text { temperature vs } \\
\text { AR post-recovery } \\
\text { ( } p=0.033 \text { ) } \\
\text { AR } \downarrow \text { BLa vs CCT } \\
\& \text { CWI } \\
\text { (AR: - } 75 \%, \text { CCT: - } \\
62 \%, \text { CWI: -62\%) } \\
\text { No sig dif RPE } \\
\text { between } \\
\text { conditions } \\
\text { No sig dif } \\
\text { HRrecovery between } \\
\text { conditions } \\
\text { ( } p=0.178 \text { ) }\end{array}$ & $\begin{array}{l}\text { CCT, CWI \& AR } \\
=\text { mean power, } \\
\text { RPE \& HR recovery } \\
\text { CWI > CCT } \downarrow \\
\text { core body } \\
\text { temperature post } \\
\text { treatment } \\
\text { CWI > AR } \downarrow \text { core } \\
\text { body } \\
\text { temperature } \\
\text { post-recovery } \\
\text { AR }>\text { CWI \& } \\
\text { CCT } \downarrow \text { BLa }\end{array}$ \\
\hline $\begin{array}{l}\text { Schniepp } \\
\text { et al, } 2002\end{array}$ & 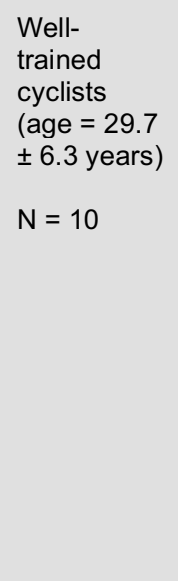 & $\begin{array}{l}\text { Pre(s1): } \\
\text { 30s sprint } \\
\text { Post(s2): } \\
\text { 30s sprint }\end{array}$ & $\begin{array}{l}\mathrm{CWI}\left(12^{\circ} \mathrm{C} \text {, hip height) }\right. \\
\text { Passive seated (CON) } \\
\text { Duration: } \\
\text { 15-mins }\end{array}$ & $\begin{array}{l}\text { PPO } \\
\text { Mean power } \\
\text { Mean HRpost- } \\
\text { recovery }\end{array}$ & $\begin{array}{l}\text { CWI } \downarrow \text { PPO vs } \\
\text { CON (CON: }-52.2 \\
\text { W [-4.7\%], CWI: - } \\
157.6 \text { W [-13.7 } \\
\%], p<0.001) \\
\text { CWI } \downarrow \text { mean } \\
\text { power vs CON } \\
\text { (CON: - } 18.4 \text { W [- } \\
2.3 \%], \text { CWI: }-76.9 \\
\text { W [-9.5\%], } p< \\
0.001) \\
\text { CWI } \downarrow \text { mean } \\
\text { HRpost-recovery vs } \\
\text { CON (CON: }+2.4 \\
\text { b-min }-1 \text { [-1 }[+1.5 \%], \\
\text { CWI: }-6.8 \text { b min }{ }^{-1} \\
[-4.2 \%], p<0.02)\end{array}$ & $\begin{array}{l}\mathrm{CON}>\mathrm{CWI} \\
\text { attenuating } \downarrow \\
\mathrm{PPO} \text { and mean } \\
\text { power } \\
\mathrm{CWI} \downarrow \text { mean } \\
\mathrm{HR}_{\text {post-recovery }}\end{array}$ \\
\hline
\end{tabular}


Road cycling events result in short resting durations and in events such as stage races, the resting location is not always the same (Chan et al. 2016). Therefore, CWI is not always practical as it would require a movable immersion pool and as a result, Chan and colleagues (Chan et al. 2016) have examined the use of a dynamic form of cold compression (Game Ready; CoolSystems, Concord, CA, USA). Results indicated that the device was no more beneficial than AR or CWI at attenuating mean power, RPE or HR following 30-mins cycling comprised of $15-\mathrm{mins}$ at $75 \% \mathrm{PPO}$ and a $15-\mathrm{min}$ maximal cycling $\mathrm{TT}$ in the heat $\left(31^{\circ} \mathrm{C}\right)$. Furthermore, AR was more beneficial than dynamic cold compression at reducing BLa measures; indicating that the use of an indoor bicycle bike roller to perform AR between events may be more effective than dynamic cold compression for enhancing recovery when an immersion pool is not practical or available.

CWI $15{ }^{\circ} \mathrm{C} \times 15$-mins has been shown beneficial for improving 1-15min TT total work performed, while 10 ${ }^{\circ} \mathrm{C}-15^{\circ} \mathrm{C}$ used for $5-14$ mins is better utilized during 5 $-15 \mathrm{~s}$ sprints for mean power output improvements and $14{ }^{\circ} \mathrm{C}$ x 5-mins can enhance subsequent $4 \mathrm{~km}$ (4-5mins) average power output and time to completion. CWI has also been shown more beneficial than AR at improving total work. While CWI was detrimental to isokinetic and isometric muscle contraction, isometric muscle testing is perhaps not a valid method of performance reporting for cyclists due to the concentric demand of cycling. These performance benefits were associated with a reduction in $\mathrm{HR}$ recovery, increased $\mathrm{HRV}$, a reduction in body temperature and increased perceived recovery. CWI may not improve perceived mental recovery, tiredness or readiness.

To better understand the role of BLa in performance from the use of CWI, future research should explore a subsequent performance bout and examine BLa pre and post recovery. Furthermore, not using a control condition confounds results as benefits can be observed from other recovery modalities, therefore, a passive seated CON condition is imperative. Recovery durations were too long in some studies and authors should implement recovery durations with greater ecological validity. To avoid limiting the impact of a recovery intervention, cyclists should not be confined to one gear during a performance trial and be allowed to dictate the load. Certainly, the pre-fatiguing exercise protocol can be controlled to ascertain the same level of fatigue in participants, however, the performance trial should not be controlled/limiting.

\section{Contrast, Thermoneutral and Hot Water Immersion/Therapy}

Contrast water therapy (CWT) can be described as brief exposure to contrasted temperature, typically ranging from $15^{\circ} \mathrm{C}$ and below for the lower range and $35^{\circ} \mathrm{C}$ and above for the upper temperature range (Table 5) (Ménétrier et al. 2013). It is proposed that CWT improves muscle soreness, inflammation and performance recovery (Vaile et al. 2008b).
Thermoneutral water immersion (TWI) can be described as exposure to temperate-water, typically around $26^{\circ} \mathrm{C}$ and has been suggested as effective in the removal of heat when exercise hyperthermia is of concern. Therefore, in order to maintain exercise performance following exercise in hot and humid conditions, TWI may be as effective as CWI (Lit et al. 2014). Indeed, it has been suggested that a reduction in muscle temperature can impair cross-bridge cycling, motor unit activation and enzyme activity rate (Schniepp et al. 2002); therefore warranting further investigation for the use of TWI.

Hot water immersion/therapy (HWI) involves immersing the body into water temperatures typically exceeding $36^{\circ} \mathrm{C}$ (Vaile et al. 2008b). Whether or not hot water immersion is beneficial to exercise recovery and performance, or the physiological mechanisms by which HWI would impact these variables are unknown (Vaile et al. 2008b).

CWT has been shown more beneficial than passive rest alone and appears dose-dependent with 6-mins shown to improve 15-min TT total work performed, where 12mins and 18-mins had no significant difference on total work performed (Versey et al. 2011). In the same study, both 6 and 12-mins improved $5 \times 15$ s sprint total work performed, where 18-mins was again ineffective (Versey et al. 2011). When examining PPO, CWT used for 12-mins was more beneficial than both 6-mins, 18mins and CON (Versey et al. 2011). When the ratio of hot immersion increased to 1:2-mins (cold:hot); 12-mins of CWI improved 5-min TT mean power by $4.1 \%$ (Ménétrier et al. 2013). Fourteen minutes of CWT improved 9-min TT mean power by up to $1.7 \%$ over 5 days and sprint cycling mean power by up to $2.2 \%$ over the same 5-day protocol (Vaile et al. 2008b). The improvement in TT mean power from CWT was more beneficial than HWI, with mean power in the HWI condition ranging from an increase of $1.5 \%$ to a reduction of $3.4 \%$ over the 5 days. When examining total work performed, CWT again, was more beneficial than HWT (Vaile et al. 2008b).

One study exhibited no improvements in time to completion or power output from the use of CWT when

compared with CON (Stanley et al. 2012). However, the performance trial in this study was based on a standardized amount of work ( $75 \%$ PPO x 15 -mins) and interestingly, authors reported an increase in power output during $40-80 \%$ of the performance trial from the use of CWT. Furthermore, the same study that reported no benefit from the use of CWT used a 190-min recovery period, which would have diluted the impact of the intervention.

Based on the evidence, CWT $\left(15^{\circ} \mathrm{C} \mathrm{CWI}: 38^{\circ} \mathrm{C} \mathrm{HWI}\right.$; $1: 1$-mins) used for 14-mins is recommended for a 9 -min TT, 6-mins is recommended for up to a $15 \mathrm{~min}$ TT, while 12 -mins appears more beneficial for a $15 \mathrm{~s}$ sprint. When the HWI:CWI ratio extended to 1-min CWI and 2-min HWI, 5-min TT total work improved. These benefits were associated with a reduction in BLa of $2.7 \mathrm{mmol} \cdot \mathrm{L}$ ${ }^{1}$ (Ménétrier et al. 2013), a decrease in perceived muscle 
Table 5. Summary of studies examining the use of contrast, thermoneutral and hot water immersion/therapy post-exercise in cyclists.

\begin{tabular}{|c|c|c|c|c|c|c|}
\hline Study & $\begin{array}{l}\text { Sample/ } \\
\text { Training } \\
\text { Status/ } \\
\text { Sample } \\
\text { Size }\end{array}$ & Exercise Protocol & $\begin{array}{l}\text { Recovery Strategy \& } \\
\text { Duration }\end{array}$ & $\begin{array}{l}\text { Markers of } \\
\text { Recoveryl } \\
\text { Performance }\end{array}$ & Results & Overall \\
\hline $\begin{array}{l}\text { Lit et al, } \\
2014\end{array}$ & $\begin{array}{l}\text { Trained } \\
\text { male } \\
\text { cyclists } \\
\text { representing } \\
\text { Kelantan } \\
\text { state }(\text { age }= \\
19 \pm 5 \\
\text { years; } \\
\mathrm{VO}_{2 \max }=58 \\
\pm 4 \mathrm{~mL} \cdot \mathrm{Kg}^{-} \\
\left.{ }^{1} \cdot \mathrm{min}^{-1}\right) \\
\mathrm{N}=9\end{array}$ & $\begin{array}{l}\text { Pre: } \\
60 \text {-mins cycling in heat @ } \\
70 \% \mathrm{VO}_{2 \max }\left(31.2 \pm 0.3^{\circ} \mathrm{C} \text {, }\right. \\
72 \pm 0.7 \% \text { rh }) \\
\text { Post: } \\
20-\mathrm{km} \text { TT }\end{array}$ & $\begin{array}{l}\text { TWI }\left(25^{\circ} \mathrm{C}\right) \\
\text { Passive rest }(\mathrm{CON})\left[25^{\circ} \mathrm{C} \text {, }\right. \\
\text { rh not described, shoulder } \\
\text { height] } \\
\text { Duration: } \\
\text { 30-mins }\end{array}$ & 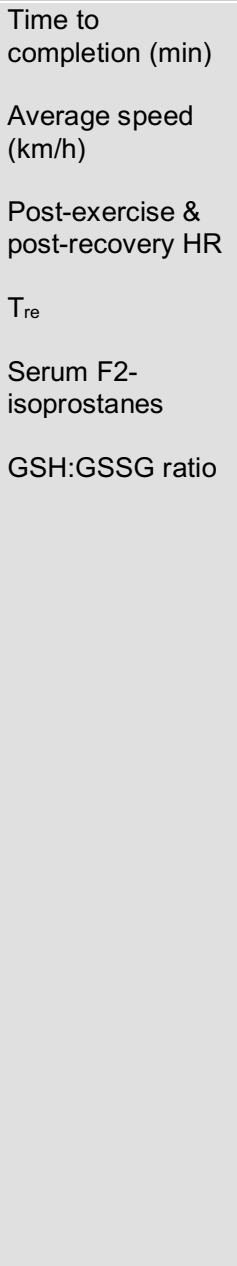 & 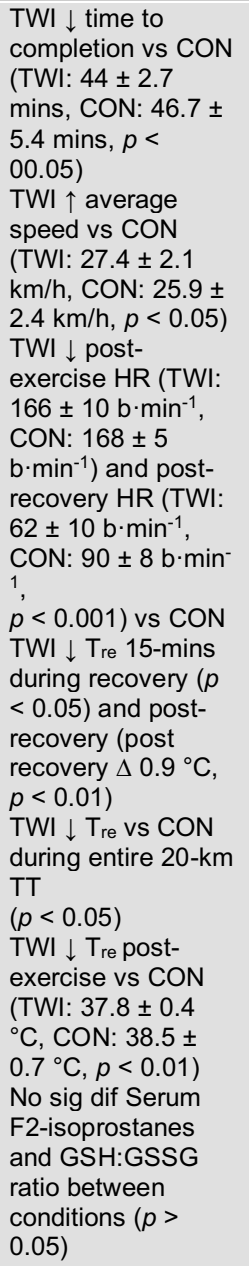 & $\begin{array}{l}\text { TWI > CON } \downarrow \text { time } \\
\text { to completion } \\
\text { TWI }>\text { CON } \uparrow \\
\text { average speed } \\
\text { TWI }>\text { CON } \downarrow \text { HR } \\
\text { TWI }>\text { CON } \downarrow \text { Tre }\end{array}$ \\
\hline $\begin{array}{l}\text { Menetrier } \\
\text { et al, } 2013\end{array}$ & $\begin{array}{l}\text { Competitive } \\
\text { male } \\
\text { cyclists } \\
(\mathrm{PPO}=5.0 \\
\pm 0.2 \mathrm{~W} / \mathrm{Kg}) \\
\mathrm{N}=12\end{array}$ & $\begin{array}{l}\text { Pre: } \\
\text { 10-min cycling } \\
\text { (5-mins 80\% PPO \& 5-mins } \\
90 \% \text { PPO) } \\
\text { Post: } \\
\text { 5-min maximal cycling }\end{array}$ & $\begin{array}{l}\text { Passive seated }\left[\sim 21^{\circ} \mathrm{C} \text {, }\right. \\
\sim 30 \% \text { rh] }(\mathrm{CON}) \\
\text { CWT }(4 \times 3 \text {-min to top thigh; } \\
\text { 1-min cold bath }\left[10-12^{\circ} \mathrm{C}\right], 2- \\
\text { min hot bath }\left[36-38^{\circ} \mathrm{C}\right], 5 \mathrm{~s} \\
\text { changeover) } \\
\mathrm{CS} \text { (according to } \\
\text { manufacturer: calf }= \\
27 \mathrm{mmHg} \text {; thigh = } 14 \mathrm{mmHg}) \\
\text { Duration: } \\
\text { 1.5-mins passive seated pre } \\
\text { and post condition } \\
\text { 12-mins per condition }\end{array}$ & $\begin{array}{l}\text { 5-min maximal } \\
\text { cycling mean } \\
\text { power } \\
\text { BLa } \\
\text { Perceived muscle } \\
\text { soreness } \\
\text { HR } \\
\text { RPE }\end{array}$ & $\begin{array}{l}\text { CWT } \uparrow \text { mean } \\
\text { power vs CON } \\
(368 \pm 12 \mathrm{~W},+4.1 \\
\pm 0.7 \% ; p<0.001) \\
\text { and } \\
\text { vs CS }(+2.2 \pm 0.8 \\
\% ; p<0.05) \\
\text { CS } \uparrow \text { mean power } \\
\text { vs CON } \\
(361 \pm 15 \mathrm{~W},+1.8 \\
\pm 1.0 \% ; p<0.05) \\
\text { CWT \& CS } \downarrow \text { BLa } \\
\text { vs CON (CWT: } 5.7 \\
\pm 1.0 \text { mmol } \cdot L^{-1} ; p< \\
0.001, \mathrm{CS}: 7.3 \pm \\
1.2 \text { mmol } \cdot \mathrm{L}^{-1} ; p< \\
0.05, \mathrm{CON}: 8.4 \pm \\
\left.1.0 \mathrm{mmol} \cdot \mathrm{L}^{-1}\right) \\
\text { CWT } \downarrow \mathrm{BLa} \text { vs CS } \\
(p<0.05) \\
\text { CWT \& CS } \downarrow \\
\text { perceived muscle } \\
\text { soreness vs CON } \\
(\mathrm{CWT}: 1.1 \pm 0.5 \text { au; } \\
p<0.001, \mathrm{CS}: 1.6 \\
\pm 0.4 \text { au; } p<0.001, \\
\text { CON: } 3.2 \pm 0.5 \text { au) } \\
\text { HR during exercise } \\
\& \text { RPE no sig dif } \\
\text { between conditions } \\
(p>0.05)\end{array}$ & $\begin{array}{l}\text { CWT \& CS > } \\
\text { CON } \uparrow \text { mean } \\
\text { power \& } \downarrow \\
\text { perceived muscle } \\
\text { soreness and BLa } \\
\text { CWT > CS } \uparrow \\
\text { mean power \& } \downarrow \\
\text { BLa } \\
\text { CWT, CS \& CON } \\
=\text { HR during } \\
\text { exercise and RPE }\end{array}$ \\
\hline
\end{tabular}




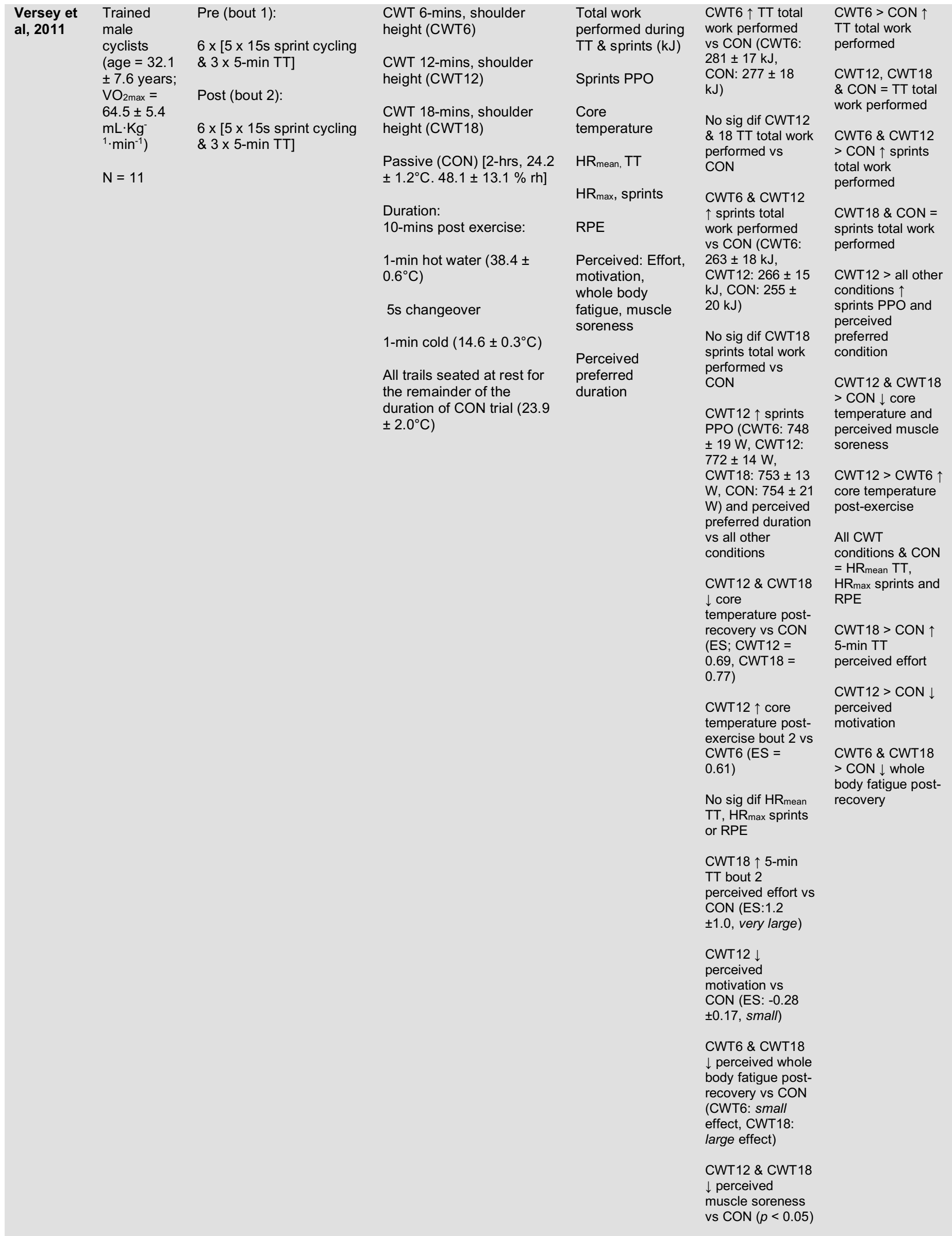




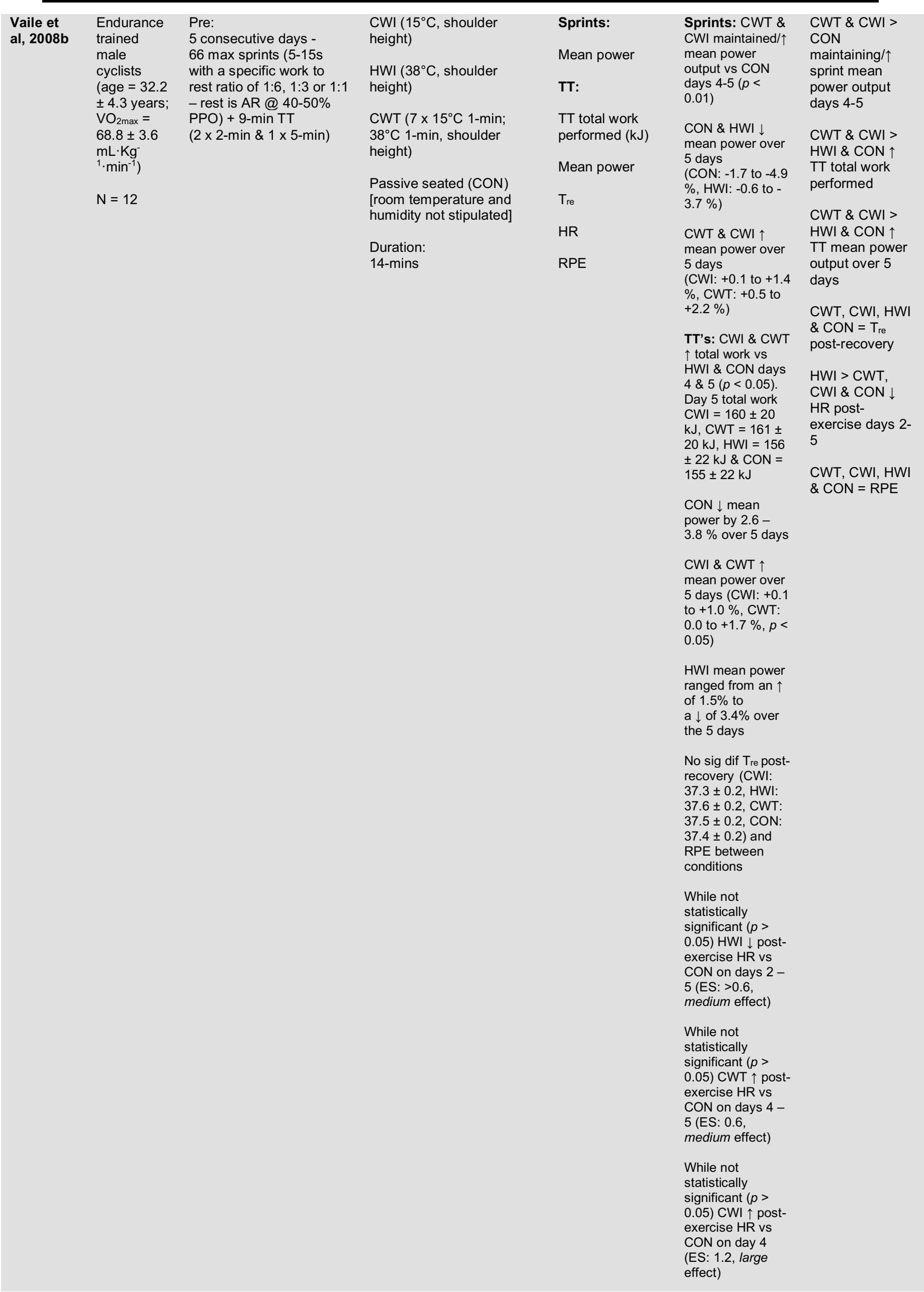




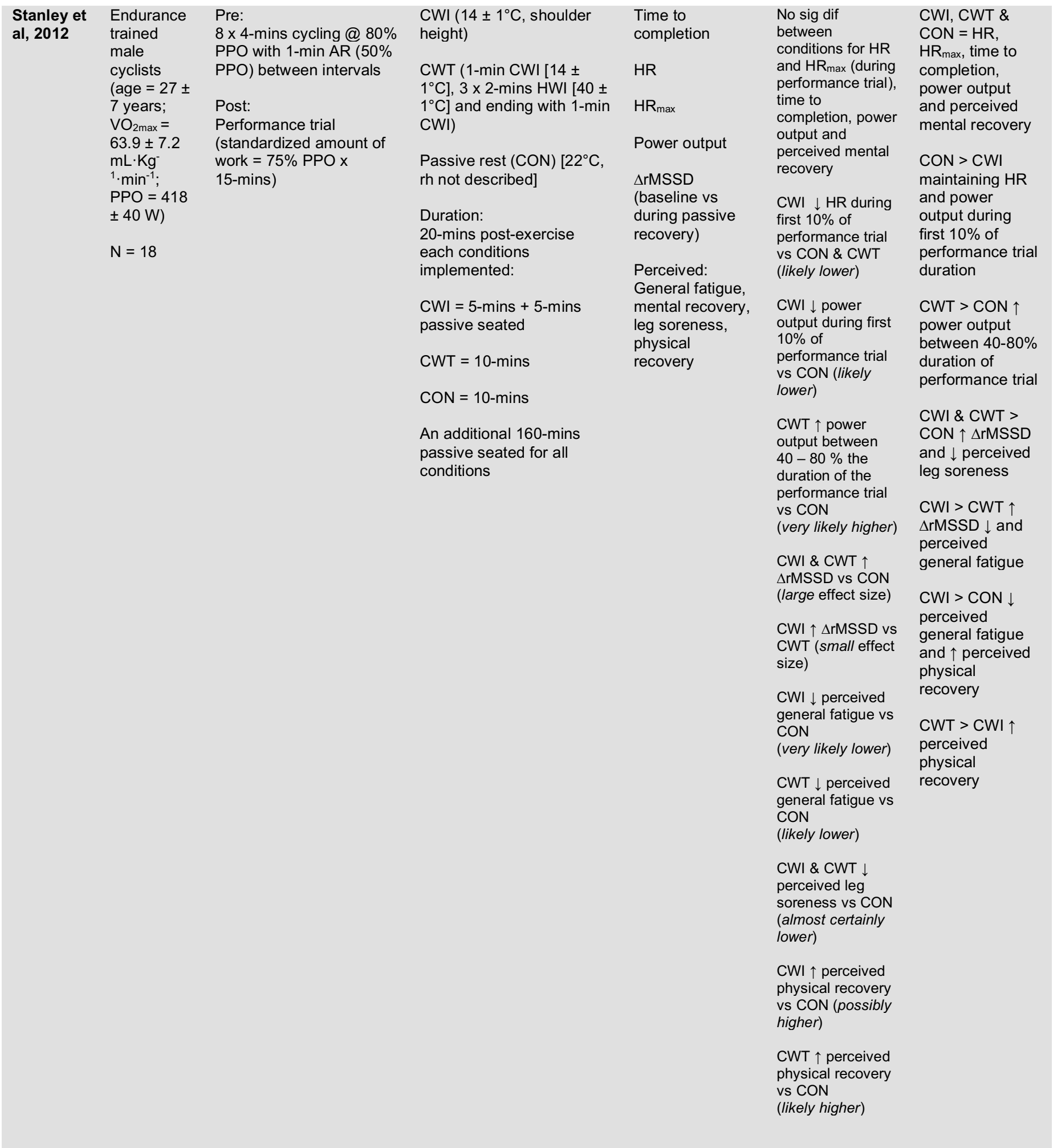

VO2max maximal oxygen uptake, N number of cyclists, rh relative humidity, TT time trial, TWI thermoneutral water immersion/therapy, CWT contrast water therapy, CWI cold water immersion, HWI hot water immersion/therapy, CON control condition/passive rest, HR heart rate, Tre rectal temperature, GSH reduced glutathione, GSSG oxidised glutathione, PPO peak power output, W/Kg watts per kilogram of bodyweight, CS compression stockings, BLa blood lactate concentration, RPE ratings of perceived exertion, W watts, HRmax maximum heart rate, AR active recovery, rMSSD natural logarithm of the square root of mean squared differences of successive $R-R$ intervals. 
soreness, whole body fatigue (Ménétrier et al. 2013; Versey et al. 2011) and core-temperature post-recovery when used for 12 \& 18-mins (Versey et al. 2011). A placebo effect may be responsible in part for the resultant performance benefits as the least effective duration (18-mins) was associated with an increase in perceived effort, while one of the most effective durations (12-mins) was reported as the perceived preferred duration in the one study that examined a doseresponse relationship (Versey et al. 2011). Surprisingly, subjects reported a reduction in perceived motivation when CWT was used for 12-mins (Versey et al. 2011).

HWI appears detrimental to mean power output and a rise of core temperature beyond $39^{\circ} \mathrm{C}$ can result in increased perceived fatigue, a reduction in exercise performance and premature exercise termination (Peiffer et al. 2008a; Vaile et al. 2008a). Therefore, a recovery strategy that aims to expose athletes to HWI alone seems counterintuitive, unless perhaps in coldweather racing, and future studies should examine the impact of weather conditions on the effectiveness of recovery methods.

TWI has been shown greater than passive rest alone at reducing $20-\mathrm{km}$ TT time to completion and improving average speed (Lit et al. 2014). This improvement in performance was associated with a reduction in $\mathrm{T}_{\mathrm{re}}$ and increased HR recovery. The use of TWI seems promising and future research should use four conditions and compare TWI, CWI, CWT and CON to determine the most effective form of water immersion.

\section{Electromyostimulation (EMS)}

Only one study to our knowledge, has examined electromyostimulation/electronic muscle stimulation (EMS) on cyclists during a cycling exercise protocol (Table 6) (Argus et al. 2013). EMS involves attaching electrodes to the skin and emitting electrical current to the muscle belly or muscle nerve in order to create small muscle contractions; it is believed that this stimulus increases blood flow, aids in the removal of metabolites, decreases muscle soreness and ultimately restores neuromuscular function and exercise performance (Babault et al. 2011). In the study by Argus and colleagues (Argus et al. 2013), participants were required to perform three bouts of 30s maximal sprint cycling, using a preload of $60 \mathrm{~s}$ cycling at $4.5 \mathrm{~W} / \mathrm{Kg}$ and 20-mins recovery between each bout. Whilst EMS was unable to significantly alter power results, a trend in BLa reduction was observed when compared with CON (4.9 $\pm 6.9 \%$ ) and EMS was able to improve participant's perceived recovery $(0.7 \pm 0.9)$. While further research is necessary to support the current findings, EMS appears to be an effective strategy at improving BLa clearance and perceptions of recovery. It should be noted that the EMS group performed the first sprinting bout at $15-20 \mathrm{~W}$ greater than the opposing conditions and therefore while results were unclear, the potential for a performance improvement may occur in future research that aims to control pre-fatigue.
Humidification Therapy (HUM)

The aforementioned study which examined EMS (Argus et al. 2013), also examined a novel strategy called Humidification Therapy (HUM) on cyclists (Table 7). HUM encompasses the delivery of high flow rates $\left(5-50 \mathrm{~L} \cdot \mathrm{min}^{-1}\right)$ of warm $\left(37^{\circ} \mathrm{C}\right)$ humidified air $(100 \%)$ through a nasal cannula, causing a low level of positive airway pressure; while speculative, it is believed that this strategy can improve the efficiency of respiratory muscles, resulting in decreased oxygen consumption and requirement, reduced BLa concentration and improved perceptions of recovery (Argus et al. 2013; Hasani et al. 2008). In the study by Argus and colleagues (Argus et al. 2013), participants were required to perform three bouts of $30 \mathrm{~s}$ maximal sprint cycling, using a preload of 60 s cycling at 4.5 $\mathrm{W} / \mathrm{Kg}$ and 20 -mins recovery between each bout. It was identified that HUM attenuated the decrement in mean power over the three exercise bouts when compared with CON $(2.2 \pm 2.5 \%)$. In conjunction with improved power measures, HUM was able to reduce BLa levels during the recovery period $(4.3 \pm 7.9 \%)$.

While further research is necessary to support the current findings, HUM appears a worthwhile tool for cyclists to increase anaerobic power measures and enhance recovery when there is a short turnaround between cycling events.

\section{Sports Massage (SM)}

Sports massage is commonly used to attenuate muscular fatigue (Bielik 2010) and it is believed that through sports massage, there is an increase in blood flow which assists in the removal of metabolic waste (Martin et al. 1998). Additionally, sports massage with ozonized oil (SMOZO) (30\% ozonized sunflower seed oil with $0.5 \%$ alpha-lipoic acid) has been shown to promote local microcirculation, cellular oxygen uptake and stimulate oxidative defensive enzymatic systems, which could further enhance recovery (Paoli et al. 2013). In the study by Paoli and colleagues (Paoli et al. 2013) SMOZO increased PPO by up to $30 \mathrm{~W}$ following anaerobic cycling when compared with SM alone and CON. Bielik and colleagues (Bielik 2010) revealed no statistically significant difference between SM and CON albeit, there was a $46 \mathrm{~W}$ difference between conditions and had an effect size analysis been conducted, perhaps an effect would have been observed. Interestingly in a study by Monedero \& Donne, a combination of both AR and SM were more effective than either SM or passive recovery alone and reduced subsequent performance time by up to $7 \mathrm{~s}$ over $5 \mathrm{~km}$ (Monedero and Donne 2000). Due to SM potential to increase the removal of metabolic waste, one would expect a consistent improvement in BLa from the use of SM. Nevertheless, results are confounding with CON shown to be more beneficial at reducing BLa 15mins post exercise (Martin et al. 1998) and AR shown to be more beneficial than SM at reducing BLa postrecovery (Table 8) (Bielik 2010; Martin et al. 1998; Monedero and Donne 2000). Consistent with performance results, both SMOZO and a combination of AR and SM, prove more effective than both SM alone 
and CON at reducing BLa (Monedero and Donne 2000; Paoli et al. 2013). Psychologically, SM both with and without ozonised oil were more beneficial than $\mathrm{CON}$ at reducing perceived fatigue (Paoli et al. 2013). Nevertheless, SMOZO was still more effective than SM alone. SM was more beneficial than AR at reducing HR measures (Bielik 2010; Monedero and Donne 2000) but also revealed no difference when compared with $\mathrm{CON}$ or SMOZO (Paoli et al. 2013). While more research is necessary to support the current findings, it appears that SM, SMOZO and a combination of AR and SM are more effective than passive rest at improving recovery, subsequent $30 \mathrm{~s}$ power output and 5-9min TT performance time.

Table 6. Summary of studies examining the use of electromyostimulation post-exercise in cyclists.

\begin{tabular}{|c|c|c|c|c|c|c|}
\hline Study & $\begin{array}{l}\text { Sample/ } \\
\text { Training } \\
\text { Status/ } \\
\text { Sample } \\
\text { Size }\end{array}$ & Exercise Protocol & $\begin{array}{l}\text { Recovery Strategy \& } \\
\text { Duration }\end{array}$ & $\begin{array}{l}\text { Markers of } \\
\text { Recoveryl } \\
\text { Performance }\end{array}$ & Results & Overall \\
\hline $\begin{array}{l}\text { Argus et } \\
\text { al, } 2013\end{array}$ & $\begin{array}{l}\text { Highly } \\
\text { trained } \\
\text { cyclists (A/B } \\
\text { grade) } \\
\mathrm{N}=11\end{array}$ & $\begin{array}{l}\text { Pre: } \\
30 \text { s max sprint cycling (S1) } \\
\text { with } 60 \text { s preload @ } \\
4.5 \mathrm{~W} / \mathrm{Kg} \\
\text { Post } 1 \text { (S2) } \\
\text { \& Post } 2 \text { (S3): } \\
30 \text { s max sprint cycling with } \\
60 \text { s preload } \\
@ 4.5 \mathrm{~W} / \mathrm{Kg}\end{array}$ & $\begin{array}{l}\text { COMP (calf: } 27 \pm 6 \mathrm{mmHg} \text {; } \\
\text { thigh: } 18 \pm 2 \mathrm{mmHg}) \\
\text { EMS }(15.7 \pm 2.8 \mathrm{~Hz}) \\
\text { HUM } \\
\text { Passive (CON) } \\
\text { Duration: } 2 \times 20-\text { mins } \\
\text { between bouts (R1 \& } 2)\end{array}$ & $\begin{array}{l}\text { 30s cycling mean } \\
\text { power } \\
\text { BLa } \\
\text { TQR } \\
\text { Belief }\end{array}$ & $\begin{array}{l}\text { COMP attenuated } \\
\downarrow \text { mean power vs } \\
\text { CON S1 - S } 2 \\
\text { (0.8 } \pm 1.2 \% \text {, } \\
\text { possibly beneficial) } \\
\& \text { S1 - S3 } \\
(1.2 \pm 1.9 \% \text {; } \\
\text { possibly beneficial) } \\
\text { HUM attenuated } \downarrow \\
\text { mean power vs } \\
\text { CON from S1 - S3 } \\
\text { (2.2 } \pm 2.5 \%, \text { likely } \\
\text { beneficial) } \\
\text { COMP no sig dif } \\
\text { BLa or TQR vs } \\
\text { CON ( } p>0.05) \\
\text { HUM \& EMS } \downarrow \text { R2 } \\
\text { BLa vs CON (HUM: } \\
4.3 \pm 7.9 \%, \\
\text { possibly beneficial, } \\
\text { EMS: } 4.9 \pm 6.9 \% \text {, } \\
\text { possibly beneficial) } \\
\text { EMS } \uparrow \text { R2 TQR vs } \\
\text { CON (0.7 } \pm 0.9, \\
\text { likely beneficial) } \\
2 \text { / } 8 \text { participants } \\
\text { accurately } \\
\text { predicted which } \\
\text { strategy would } \\
\text { enhance their } \\
\text { recovery (belief). }\end{array}$ & $\begin{array}{l}\text { COMP \& HUM > } \\
\text { CON attenuating } \\
\downarrow \text { mean power } \\
\text { COMP \& CON = } \\
\text { BLa \& TQR } \\
\text { HUM \& EMS > } \\
\text { CON } \downarrow \text { BLa } \\
\text { EMS > CON } \uparrow \\
\text { TQR } \\
\text { Possibly no } \\
\text { placebo effect } \\
\text { (2/8 belief) }\end{array}$ \\
\hline
\end{tabular}

$N$ number of cyclists, W/Kg watts per kilogram of bodyweight, COMP compression garments/full length tights, EMS electromyostimulation/electronic muscle stimulation, HUM humidification therapy, CON control condition/passive rest, R1 \& 2 recovery one and recovery two, BLa blood lactate concentration, TQR perceived total quality recovery. 
Table 7. Summary of studies examining the use of humidification therapy post-exercise in cyclists.

\begin{tabular}{|c|c|c|c|c|c|c|}
\hline Study & $\begin{array}{l}\text { Sample/ } \\
\text { Training } \\
\text { Status/ } \\
\text { Sample } \\
\text { Size }\end{array}$ & Exercise Protocol & $\begin{array}{l}\text { Recovery Strategy \& } \\
\text { Duration }\end{array}$ & $\begin{array}{l}\text { Markers of } \\
\text { Recoveryl } \\
\text { Performance }\end{array}$ & Results & Overall \\
\hline $\begin{array}{l}\text { Argus et } \\
\text { al, } 2013\end{array}$ & $\begin{array}{l}\text { Highly } \\
\text { trained } \\
\text { cyclists (A/B } \\
\text { grade) } \\
\mathrm{N}=11\end{array}$ & $\begin{array}{l}\text { Pre: } \\
\text { 30s max sprint cycling (S1) } \\
\text { with } 60 \text { s preload @ } \\
4.5 \mathrm{~W} / \mathrm{Kg} \\
\text { Post } 1 \text { (S2) } \\
\text { \& Post } 2 \text { (S3): } \\
\text { 30s max sprint cycling with } \\
60 \text { s preload } \\
\text { @ } 4.5 \mathrm{~W} / \mathrm{Kg}\end{array}$ & $\begin{array}{l}\text { COMP (calf: } 27 \pm 6 \mathrm{mmHg} \text {; } \\
\text { thigh: } 18 \pm 2 \mathrm{mmHg} \text { ) } \\
\text { EMS }(15.7 \pm 2.8 \mathrm{~Hz}) \\
\text { HUM } \\
\text { Passive (CON) } \\
\text { Duration: } 2 \times 20 \text {-mins } \\
\text { between bouts (R1 \& 2) }\end{array}$ & $\begin{array}{l}\text { 30s cycling mean } \\
\text { power } \\
\text { BLa } \\
\text { TQR } \\
\text { Belief }\end{array}$ & 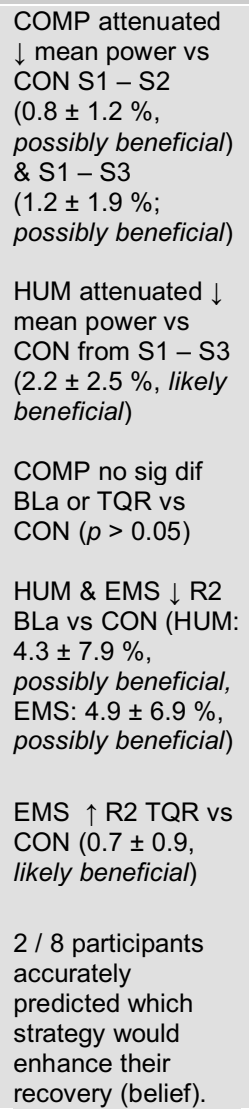 & $\begin{array}{l}\text { COMP \& HUM > } \\
\text { CON attenuating } \\
\downarrow \text { mean power } \\
\text { COMP \& CON = } \\
\text { BLa \& TQR } \\
\text { HUM \& EMS > } \\
\text { CON } \downarrow \text { BLa } \\
\text { EMS > CON } \uparrow \\
\text { TQR } \\
\text { Possibly no } \\
\text { placebo effect } \\
\text { (2/8 belief) }\end{array}$ \\
\hline
\end{tabular}

$N$ number of cyclists, W/Kg watts per kilogram of bodyweight, COMP compression garments/full length tights, EMS electromyostimulation/electronic muscle stimulation, HUM humidification therapy, CON control condition/passive rest, R1 \& 2 recovery one and recovery two, BLa blood lactate concentration, TQR perceived total quality recovery.

\section{Static Stretching (SS)}

To our knowledge, the current research evaluating static stretching (SS) on cyclists is limited to one study (Table 9) (Kingsley et al. 2013). SS, while beneficial for increasing range of motion (RoM), has been shown to temporarily decrease muscular power (Costa et al. 2013; Samuel et al. 2008). In the study by Kingsley and colleagues (Kingsley et al. 2013), SS resulted in no significant difference for any of the performance

variables measured when compared with quiet rest (QR). Unfortunately, the details of how QR was performed were not described. While no significant difference was observed, SS resulted in a $0.86 \%$ increase in absolute $\mathrm{PPO}$ and increased relative peak power output $(+0.86 \%)$ when compared with QR. The use of Cohen's $d$ effect size analysis would have been a worthwhile tool to better evaluate the findings of the study. As expected, SS improved RoM and resulted in a $2.1 \mathrm{~cm}$ increase in sit and reach distance. With limited research, it is difficult to interpret the efficacy of SS. However, based on the aforementioned study, it can be deduced that SS does not inhibit anaerobic cycling power if used for $3 \times 30$ s per muscle and is a worthwhile inclusion where RoM is limited and an increase in RoM will prove advantageous to performance. Indeed, cycling has been linked to increased quadriceps muscle group, hamstrings muscle group and ITB tightness; which have been suggested to increase force on the knee and the potential for injury (Asplund and St Pierre 2004). Therefore, performing quadriceps, hamstring and ITB stretching between exercise bouts could be beneficial.

\section{Journal of Science and Cycling}


Table 8. Summary of studies examining the use of sports massage post-exercise in cyclists.

\begin{tabular}{|c|c|c|c|c|c|c|}
\hline Study & $\begin{array}{l}\text { Sample/ } \\
\text { Training } \\
\text { Status/ } \\
\text { Sample } \\
\text { Size }\end{array}$ & Exercise Protocol & $\begin{array}{l}\text { Recovery Strategy \& } \\
\text { Duration }\end{array}$ & $\begin{array}{l}\text { Markers of } \\
\text { Recoveryl } \\
\text { Performance }\end{array}$ & Results & Overall \\
\hline $\begin{array}{l}\text { Bielik, } \\
2010\end{array}$ & $\begin{array}{l}\text { Junior elite } \\
\text { Slovakian } \\
\text { off-road } \\
\text { cyclists (age } \\
=19 \pm 1 \\
\text { years; } \\
\mathrm{VO}_{2 \mathrm{max}}=67 \\
\pm 3 \mathrm{~mL} \cdot \mathrm{Kg}^{-} \\
{ }^{1} \cdot \mathrm{min}^{-1} \text { ) } \\
\mathrm{N}=11\end{array}$ & $\begin{array}{l}\text { Pre: } \\
3 \times 30 \text { s WAnT (s1-3) with } \\
4-\text { min recovery between } \\
\text { intervals } \\
\text { Post: } \\
30 \text { s WAnT (s4) }\end{array}$ & $\begin{array}{l}\text { Passive recovery (CON) } \\
\text { SM } \\
\text { AR (10-mins @ 20\% VO } 2 \text { max } \\
\text { and } 10 \text {-mins } \\
\text { @ 40\% VO } \text { max }^{\text {max }} \\
\text { Duration: } \\
\text { 20-mins }\end{array}$ & $\begin{array}{l}\text { PPO } \\
\text { Mean power } \\
\text { Fatigue index \% } \\
\text { BLa } \\
\text { HR }_{\text {recovery }}\end{array}$ & 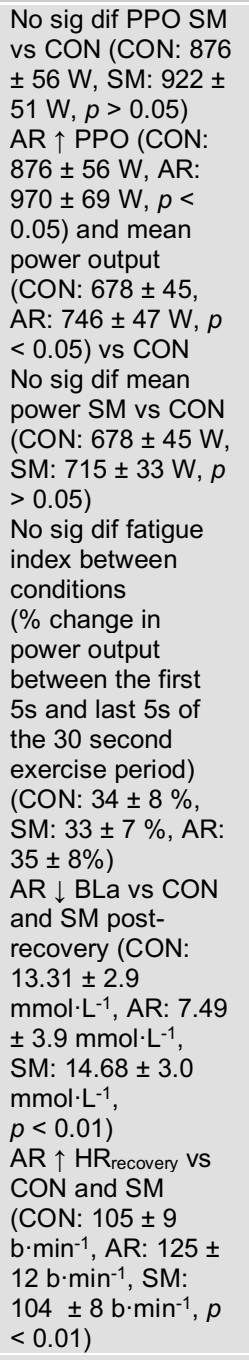 & $\begin{array}{l}\mathrm{AR}>\mathrm{CON} \uparrow \mathrm{PPO} \\
\& \text { mean power } \\
\text { AR }>\text { CON \& SM } \\
\downarrow \text { BLa post- } \\
\text { recovery } \\
\text { AR }>\text { CON and } \\
\text { SM } \uparrow \mathrm{HR}_{\text {recovery }}\end{array}$ \\
\hline $\begin{array}{l}\text { Paoli et al, } \\
2013\end{array}$ & $\begin{array}{l}\text { Male } \\
\text { competitive } \\
\text { amateur } \\
\text { cyclists (age } \\
=27 \pm 3.5 \\
\text { years; } \\
\text { training } \\
\text { years = } 8 \pm \\
4 \text { years) } \\
\mathrm{N}=15\end{array}$ & $\begin{array}{l}\text { Pre: } \\
3 \times 30 \text { s WAnT with 2-mins } \\
\text { recovery between intervals } \\
\text { Post: } \\
\text { Ramp test until voluntary } \\
\text { termination }(3-\mathrm{min} \text { baseline } \\
\text { cycling @ } 60 \mathrm{~W}+30 \mathrm{~W} \cdot \mathrm{min}^{-} \\
1 \uparrow \text { thereafter) }\end{array}$ & $\begin{array}{l}\text { Passive rest (CON) } \\
\text { Sports massage with } \\
\text { Bioperoxoil (SMOZO) [30\% } \\
\text { ozonised sunflower seed oil } \\
\text { with } 0.5 \% \text { alpha-lipoic acid] } \\
\text { Sports massage (SM) } \\
\text { Duration: } \\
5 \text {-mins passive seated on } \\
\text { bike followed by } \\
\text { 16-mins per condition ( } 8- \\
\text { min prone and } \sim 8 \text {-min } \\
\text { supine for all conditions) }\end{array}$ & $\begin{array}{l}\text { BLa } \\
\text { HR } \text { recovery } \\
\text { Ramp test PPO } \\
\text { Perceived fatigue }\end{array}$ & $\begin{array}{l}\text { SMOZO } \downarrow \text { BLa vs } \\
\text { SM \& CON } 13- \\
\text { mins post exercise } \\
\text { when compared } \\
\text { with immediately } \\
\text { post-exercise } \\
\text { (SMOZO: }-34.3 \% \text {, } \\
\text { SM: }-22.5 \% \text {, CON: } \\
-25.4 \% \text { ) and at } 20- \\
\text { mins when } \\
\text { compared with } 13- \\
\text { mins post exercise } \\
\text { (SMOZO: }-27.6 \% \text {, } \\
\text { SM: }-27.2 \% \text {, } \\
\text { CON: }-23.2 \%) \\
\text { No sig dif HRrecovery } \\
\text { between conditions } \\
\text { ( } p>0.05) \\
\text { SMOZO } \uparrow \text { PPO vs } \\
\text { SM \& CON } \\
\text { (SMOZO: } 370 \pm 60 \\
\text { W, SM: } 340 \pm 55 \\
\text { W, CON: } 344 \pm 56 \\
\text { W, } p<0.05) \\
\text { SMOZO \& SM } \downarrow \\
\text { perceived fatigue } \\
\text { vs CON } \\
\text { ( } p<0.033 \text { ) } \\
\text { SMOZO } \downarrow \\
\text { perceived fatigue } \\
\text { vs SM } \\
(p<0.033)\end{array}$ & $\begin{array}{l}\text { SMOZO > SM \& } \\
\text { CON } \downarrow \text { BLa } \\
\text { SMOZO, SM \& } \\
\text { CON }=\text { HR } \\
\text { SMOZO > SM \& } \\
\text { CON } \uparrow \text { PPO } \\
\text { SM with and } \\
\text { without Ozonised } \\
\text { oil }>\text { CON } \downarrow \\
\text { perceived fatigue } \\
\text { SMOZO }>\text { SM } \downarrow \\
\text { perceived fatigue }\end{array}$ \\
\hline
\end{tabular}




\begin{tabular}{|c|c|c|c|c|c|c|}
\hline $\begin{array}{l}\text { Martin et } \\
\text { al, } 1998\end{array}$ & $\begin{array}{l}\text { Competitive } \\
\text { male } \\
\text { cyclists (age } \\
=24.5 \pm \\
3.98 \text { years; } \\
\mathrm{VO}_{2 \max }= \\
55.87 \pm \\
3.82 \mathrm{~mL} \cdot \mathrm{Kg}^{-} \\
{ }^{-} \cdot \mathrm{min}^{-1} \text { ) } \\
\mathrm{N}=10\end{array}$ & $\begin{array}{l}\text { Pre: } \\
3 \times 30 \text { s WAnT with 2-mins } \\
\text { passive rest between } \\
\text { intervals }\end{array}$ & $\begin{array}{l}\text { Sport massage }(\mathrm{SM}) \\
\left.\text { AR (80rpm @ } 40 \% \mathrm{VO}_{2 \max }\right) \\
\text { Passive lying in a supine } \\
\text { position (CON) } \\
\text { Duration: } \\
20 \text {-mins }\end{array}$ & BLa & $\begin{array}{l}\text { AR significantly } \downarrow \\
\text { BLa post-recovery } \\
\text { vs SM \& CON } \\
\text { (AR: }-59.38 \% \text {, } \\
\text { SM: }-36.21 \% \text {, } \\
\text { CON: }-38.67 \%) \\
\text { CON } \downarrow \text { BLa vs SM } \\
15 \text {-mins post } \\
\text { exercise }(p<0.05) \\
\text { but not at } 20 \text { or } 25- \\
\text { mins }\end{array}$ & $\begin{array}{l}\text { AR > SM \& CON } \\
\downarrow \text { BLa } \\
\text { CON > SM } \downarrow \text { BLa } \\
15 \text {-mins post } \\
\text { exercise }\end{array}$ \\
\hline $\begin{array}{l}\text { Monedero } \\
\text { \& Donne, } \\
2000\end{array}$ & $\begin{array}{l}\text { Trained } \\
\text { male } \\
\text { cyclists (age } \\
=25 \pm 1 \\
\text { years; } \\
\mathrm{VO}_{2 \max }=68 \\
\pm 1.7 \\
\mathrm{~mL} \cdot \mathrm{Kg}^{-} \\
{ }^{1} \cdot \mathrm{min}^{-1} ; \mathrm{PPO} \\
=364 \pm 9 \\
\mathrm{~W} \text {; training } \\
\text { years }=5 \pm \\
0.3 \text { years) } \\
\mathrm{N}=18\end{array}$ & $\begin{array}{l}\text { Pre \& post: } \\
\text { 5-km maximal effort } \\
\text { cycling test }\end{array}$ & $\begin{array}{l}\text { Passive seated at rest } \\
(\mathrm{CON}) \\
\text { AR }\left(50 \% \mathrm{VO}_{2 \text { max }}\right) \\
\text { SM (lower leg) } \\
\text { Combined [AR \& SM] } \\
\left(3.75 \text { min } \mathrm{AR} \mathrm{@} 50 \% \mathrm{VO}_{2 \max }\right. \\
\text { pre and post-SM, } 7.5 \text { min } \\
\text { SM) } \\
\text { Duration: } \\
\text { 15-mins }\end{array}$ & $\begin{array}{l}\text { 5-km } \\
\text { performance time } \\
\text { BLa } \\
\text { HR }_{\text {recovery }}\end{array}$ & $\begin{array}{l}\text { Combined } \\
\text { attenuated } \downarrow \\
\text { performance time } \\
\text { vs CON, AR \& SM } \\
\text { (performance time } \\
\text { increase between } \\
1^{\text {st }} \text { and } 2^{\text {nd }} \text { test; } \\
\text { CON: } 9.9 \pm 1.6 \\
\text { seconds, AR: } 6.9 \pm \\
1.3 \text { seconds, SM: } \\
7.7 \pm 1.5 \text { seconds, } \\
\text { combined: } 2.9 \pm \\
1.5 \text { seconds, } \\
p<0.01 \text { ) } \\
\text { Combined } \downarrow \text { BLa } \\
\text { vs CON \& SM ( } p< \\
0.01 \text { ) } \\
\text { coN, SM \& SM } \\
\text { portion of } \\
\text { combined } \downarrow \\
\text { HRrecovery V AR \& } \\
\text { AR portion of } \\
\text { combined during } \\
\text { recovery ( } p<0.05 \text { ) }\end{array}$ & $\begin{array}{l}\text { Combined > } \\
\text { CON, AR \& SM } \\
\text { attenuating } \downarrow 5 \mathrm{~km} \\
\text { performance time } \\
\text { Combined \& AR } \\
>\text { CON \& SM } \downarrow \\
\text { BLa } \\
\text { CON \& SM > AR } \\
\downarrow \text { HR } \\
\text { recovery }\end{array}$ \\
\hline
\end{tabular}

$V_{2 \max }$ maximal oxygen uptake, $N$ number of cyclists, WAnT wingate anaerobic cycling test, SM sports massage, $A R$ active recovery, $P P O$ peak power output, $B L a$ blood lactate concentration, $H R$ heart rate, CON control condition/passive rest, SMOZO sports massage with ozonised oil, $W$ watts

\section{Active Recovery (AR)}

Active recovery can be described as gentle exercise between exercise bouts; believed to enhance metabolic waste removal and improve subsequent performance (Chan et al. 2016). It comes as no surprise that AR increases HR to a great degree than passive rest during recovery and this increase in HR, may be one of the contributing factors as to why AR is beneficial to postexercise recovery (Bielik 2010; Monedero and Donne 2000). It is theorised that an increase in HR, concomitant increase in blood flow and metabolic rate, are all factors which lead to improved recovery and performance (Bielik 2010). With varying methods used in cycling literature (Table 11), it is difficult to discern the optimal exercise intensity and duration for improving subsequent cycling performance (Table 10). Connolly and colleagues (Connolly et al. 2003) discovered that AR used for 3-mins following 15 s sprint cycling and repeated 6 times, resulted in an attenuation of the decrement in mean power when compared with CON. The use of $\mathrm{AR}$ in an anaerobic setting was further supported by Bielik and colleagues (Bielik 2010) who identified that AR following $3 \times 30$ s WAnT with 4-min recovery between intervals was able to significantly increase PPO (CON: $876 \pm 56 \mathrm{~W}$, AR: $970 \pm 69 \mathrm{~W})$ and mean power output (CON: $678 \pm 45$, AR: $746 \pm 47 \mathrm{~W}$ ) in the following 30 s cycling WAnT. The ability for AR to attenuate a decrement in subsequent performance is not limited to anaerobic power and has been shown beneficial when implemented between $5-\mathrm{km}$ TT cycling bouts (Monedero and Donne 2000). Unfortunately, further studies examining AR in cycling either did not use a passive control and compared AR against CWI, or they simply did not examine a subsequent performance bout (Chan et al. 2016; Martin et al. 1998; Vaile et al. 2008a; Vaile et al. 2011). Comparing against CWI is difficult to interpret, as CWI has been shown to improve subsequent performance when compared with passive rest (Peiffer et al. 2008a; Stanley et al. 2013; Vaile et al. 2008b).

AR was able to attenuate BLa concentration by $21-54 \%$ more than that of CON (Bielik 2010; Martin et al. 1998; Monedero and Donne 2000). However, one study revealed no significant difference in BLa levels following AR (Connolly et al. 2003) and this could have been due to a shorter recovery duration of only 3 -min intervals (Connolly et al. 2003). The authors from this study hypothesised that perhaps measuring plasma lactate concentration as opposed to intracellular lactate concentration was not an effective method of assessing BLa given the short rest duration. A novel form of AR has been examined by performing active recovery in 
Table 9. Summary of studies examining the use of static stretching post-exercise in cyclists.

\begin{tabular}{|c|c|c|c|c|c|c|}
\hline Study & $\begin{array}{l}\text { Sample/ } \\
\text { Training } \\
\text { Status/ } \\
\text { Sample } \\
\text { Size }\end{array}$ & Exercise Protocol & $\begin{array}{l}\text { Recovery Strategy \& } \\
\text { Duration }\end{array}$ & $\begin{array}{l}\text { Markers of } \\
\text { Recoveryl } \\
\text { Performance }\end{array}$ & Results & Overall \\
\hline $\begin{array}{l}\text { Kingsley et } \\
\text { al, } 2013\end{array}$ & $\begin{array}{l}\text { Aerobically } \\
\text { trained } \\
\text { cyclists (age } \\
=21 \pm 2 \\
\text { years; } \\
\mathrm{VO}_{2 \max }= \\
42.0 \pm 5.6 \\
\mathrm{~mL} \cdot \mathrm{Kg}^{-} \\
{ }^{1} \cdot \mathrm{min}^{-1} \text { ) } \\
\mathrm{M}=9 \\
\mathrm{~F}=4\end{array}$ & $\begin{array}{l}\text { Pre: } \\
\text { 30-min cycling @ 65\% } \\
\text { VO }_{2 \max } \\
\text { Post: } \\
\text { 30s WAnT }\end{array}$ & $\begin{array}{l}\text { SS }(3 \times 30 \text { s per leg: } \\
\text { Hamstrings, quadriceps, hip } \\
\text { flexors and extensors \& } \\
\text { piriformis) } \\
\text { QR (details not described) } \\
\text { Duration: } \\
\text { 15-mins }\end{array}$ & $\begin{array}{l}\text { Sit \& reach } \\
\text { Absolute PPO } \\
\text { Relative PPO } \\
\text { RPM }_{\text {peak }}\end{array}$ & $\begin{array}{l}\mathrm{SS} \uparrow \text { Sit } \& \text { reach } \\
\text { from } 25.2 \pm 2.2 \mathrm{~cm} \\
\text { to } 27.3 \pm 1.7 \mathrm{~cm} \mathrm{(} p \\
<0.05) \\
\text { No sig dif between } \\
\text { conditions for any } \\
\text { performance } \\
\text { variable }(p>0.05) \\
\text { SS } \uparrow \text { absolute PPO } \\
\text { vs QR but no sig dif } \\
(+0.86 \%, p>0.05) \\
\text { SS } \uparrow \text { relative PPO } \\
\text { vs QR but no sig dif } \\
(+0.86 \%, p>0.05) \\
\text { SS } \uparrow R P M \text { peak vs } \\
\text { QR but no sig dif } \\
+1.90 \%, p>0.05)\end{array}$ & $\begin{array}{l}\text { SS \& QR = } \\
\text { Absolute PPO, } \\
\text { relative PPO \& } \\
\text { RPM }_{\text {peak }}\end{array}$ \\
\hline
\end{tabular}

$V_{O_{2 m a x}}$ maximal oxygen uptake, WAnT wingate anaerobic cycling test, SS static stretching, QR quiet rest, $P P O$ peak power output, $R P M$ cycling revolutions per minute.

water (ARW) (Ferreira et al. 2011). Results indicated that ARW was more effective than passive recovery on land (PRL) and passive recovery in water (PRW) at reducing $\mathrm{BLa}$ concentration 15-60mins during recovery. Additionally, there was no change in HRV between conditions however, when examining shorter resting protocols of up to 30-mins between exercise bouts, PRW and PRL appear more effective than ARW at improving HRV. Unfortunately no performance variables were examined.

The use of AR at 80RPM for 3-mins may improve $15 \mathrm{~s}$ sprint cycling power output, $\mathrm{AR}$ at $50 \% \mathrm{VO}_{2 \max }$ for 15 -mins can improve $5 \mathrm{~km}$ TT performance time and $20-40 \% \mathrm{VO}_{2 \max }$ for 20-mins can improve $30 \mathrm{~s}$ peak and mean power output. Future research should ensure that a passive rest control condition is used and that subsequent performance is examined, to support the current body of evidence. ARW is a novel recovery strategy that warrants further research. Future studies should compare ARW with $\mathrm{AR}$ on land and examine exercise performance in conjunction with physiological variables.
Table 10. Different exercise intensities and durations utilised during active recovery studies.

\begin{tabular}{|c|c|c|c|c|}
\hline Author & Intensity & Duration & $\begin{array}{l}\text { Control } \\
\text { Condition }\end{array}$ & $\begin{array}{l}\text { Subsequent } \\
\text { Performance }\end{array}$ \\
\hline $\begin{array}{l}\text { Connolly } \\
\text { et al., } \\
2003\end{array}$ & $\begin{array}{c}\text { 80rpm } \\
(1 \mathrm{Kg} \\
\text { resistance) }\end{array}$ & 3-mins & Yes & + \\
\hline $\begin{array}{l}\text { Joanna } \\
\text { Vaile et } \\
\text { al., 2008a }\end{array}$ & $\begin{array}{c}40 \% \\
\mathrm{VO}_{2 \max }\end{array}$ & 15-mins & No & - \\
\hline $\begin{array}{l}\text { Vaile et } \\
\text { al., } 2011\end{array}$ & $40 \% \mathrm{PPO}$ & 15-mins & No & - \\
\hline $\begin{array}{l}\text { Chan et } \\
\text { al., } 2016\end{array}$ & $40 \%$ PPO & 15-mins & No & $=$ \\
\hline $\begin{array}{l}\text { Monedero } \\
\text { \& Donne, } \\
2000\end{array}$ & $\begin{array}{c}50 \% \\
\mathrm{VO}_{2 \max }\end{array}$ & 15-mins & Yes & + \\
\hline $\begin{array}{l}\text { Martin et } \\
\text { al., } 1998\end{array}$ & $\begin{array}{c}40 \% \\
\mathrm{VO}_{2 \max } / \\
80 \mathrm{rpm}\end{array}$ & 20-mins & Yes & $\mathrm{n} / \mathrm{a}$ \\
\hline $\begin{array}{l}\text { Bielik, } \\
2010\end{array}$ & $\begin{array}{c}20 \% \\
\mathrm{VO}_{2 \max } \\
40 \% \\
\mathrm{VO}_{2 \max }\end{array}$ & $\begin{array}{l}\text { 10-mins } \\
10-\text { mins }\end{array}$ & Yes & + \\
\hline
\end{tabular}

+ Positive/enhanced, $=$ no change, - negative/detrimental, n/a not measured/not applicable. 
Table 11. Summary of studies examining the use of active recovery post-exercise in cyclists.

\begin{tabular}{|c|c|c|c|c|c|c|}
\hline Study & $\begin{array}{l}\text { Sample/ } \\
\text { Training } \\
\text { Status/ } \\
\text { Sample Size }\end{array}$ & Exercise Protocol & $\begin{array}{l}\text { Recovery Strategy \& } \\
\text { Duration }\end{array}$ & $\begin{array}{l}\text { Markers of } \\
\text { Recovery/ } \\
\text { Performance }\end{array}$ & Results & Overall \\
\hline $\begin{array}{l}\text { Connolly } \\
\text { et al, } 2003\end{array}$ & $\begin{array}{l}\text { Recreationally } \\
\text { active male } \\
\text { cyclists (age } \\
=21.8 \pm 3.3 \\
\text { years) }\end{array}$ & $\begin{array}{l}\text { Pre \& Post: } \\
6 \text { x } 15 \text { s sprint cycling with } \\
\text { recovery protocol between } \\
\text { intervals }\end{array}$ & $\begin{array}{l}\text { AR }(80 \mathrm{rpm} @ 1 \mathrm{Kg} \\
\text { resistance }) \times 3-\mathrm{mins} \\
\text { Passive seated on bike } \\
(\mathrm{CON}) \times 2.50 \mathrm{~s}\end{array}$ & $\begin{array}{l}\text { Mean PPO } \\
\text { Mean power } \\
\text { BLa }\end{array}$ & $\begin{array}{l}\text { Mean power no } \\
\text { sig dif between } \\
\text { conditions }(p= \\
0.57) \\
\\
\text { BLa no sig dif } \\
\text { between } \\
\text { conditions (AR: } \\
9.09 \pm 2.37 \\
\mathrm{mmol} \cdot \mathrm{L}^{-1}, \mathrm{CON} \text { : } \\
10.05 \pm 2.84 \\
\mathrm{mmol} \cdot \mathrm{L}^{-1} ; p= \\
0.37 \text { ) }\end{array}$ & $\begin{array}{l}\text { AR }>\text { CON } \\
\text { attenuating } \downarrow \\
\text { mean PPO } \\
\text { AR \& CON = } \\
\text { mean power \& } \\
\text { BLa }\end{array}$ \\
\hline \multirow{5}{*}{$\begin{array}{l}\text { Bielik, } \\
2010\end{array}$} & \multirow{5}{*}{$\begin{array}{l}\text { Junior elite } \\
\text { Slovakian off- } \\
\text { road cyclists } \\
\text { (age }=19 \pm 1 \\
\text { years; } \mathrm{VO}_{2 \max } \\
=67 \pm 3 \\
\mathrm{~mL} \cdot \mathrm{Kg}^{-1} \cdot \mathrm{min}^{-1} \text { ) }\end{array}$} & \multirow{5}{*}{$\begin{array}{l}\text { Pre: } \\
3 \times 30 \text { s WAnT (s } 1-3) \text { with } \\
4 \text {-min recovery between } \\
\text { intervals } \\
\text { Post: } \\
30 \text { s WAnT } \\
\text { (s4) }\end{array}$} & $\begin{array}{l}\mathrm{VO}_{2 \max } \text { and } 10-\operatorname{mins} @ \\
\left.40 \% \mathrm{VO}_{2 \max }\right)\end{array}$ & BLa & \multirow{2}{*}{$\begin{array}{l}\text { No sig dif PPO } \\
\text { (CON: } 876 \pm 56 \\
\text { W, SM: } 922 \pm 51 \\
\text { W, } \\
p>0.05 \text { ) and } \\
\text { mean power } \\
\text { (CON: } 678 \pm 45 \\
\text { W, } \\
\text { SM: } 715 \pm 33 \text { W, } \\
p>0.05) \text { SM vs } \\
\text { CON }\end{array}$} & $\begin{array}{l}\downarrow \text { BLa post- } \\
\text { recovery }\end{array}$ \\
\hline & & & $\begin{array}{l}\text { Duration: } \\
\text { 20-mins }\end{array}$ & $\mathrm{HR}_{\text {recovery }}$ & & $\begin{array}{l}\mathrm{AR}>\mathrm{CON} \text { and } \\
\mathrm{SM} \uparrow \mathrm{HR}_{\text {recovery }}\end{array}$ \\
\hline & & & & & $\begin{array}{l}\text { AR } \uparrow \text { PPO (CON: } \\
876 \pm 56 \mathrm{~W}, \mathrm{AR}: \\
970 \pm 69 \mathrm{~W}, \\
p<0.05) \text { and } \\
\text { mean power } \\
\text { output (CON: } 678 \\
\pm 45, \text { AR: } 746 \pm \\
47 \mathrm{~W}, p<0.05) \text { vs } \\
\text { CON }\end{array}$ & \\
\hline & & & & & $\begin{array}{l}\mathrm{AR} \downarrow \mathrm{BLa} \text { vs CON } \\
\text { and SM post- } \\
\text { recovery (CON: } \\
13.31 \pm 2.9 \\
\mathrm{mmol} \cdot \mathrm{L}^{-1}, \mathrm{AR}: \\
7.49 \pm 3.9 \\
\mathrm{mmol} \cdot \mathrm{L}^{-1}, \mathrm{SM}: \\
14.68 \pm 3.0 \\
\mathrm{mmol} \cdot \mathrm{L}^{-1}, p< \\
0.01)\end{array}$ & \\
\hline & & & & & $\begin{array}{l}\text { AR } \uparrow H R_{\text {recovery }} \text { vs } \\
\text { CON and SM } \\
\text { (CON: } 105 \pm 9 \\
\text { b.min }{ }^{-1}, A R: 125 \pm \\
12 \mathrm{~b} \cdot \mathrm{min}^{-1}, \mathrm{SM}^{-} \\
104 \pm 8 \mathrm{~b} \cdot \mathrm{min}^{-1}, p \\
<0.01 \text { ) }\end{array}$ & \\
\hline
\end{tabular}




\begin{tabular}{|c|c|c|c|c|c|c|}
\hline $\begin{array}{l}\text { Martin et } \\
\text { al, } 1998\end{array}$ & $\begin{array}{l}\text { Competitive } \\
\text { male } \\
\text { cyclists } \\
(\text { age }=24.5 \\
\pm 3.98 \\
\text { years; } \\
\mathrm{VO}_{2 \max }= \\
55.87 \pm \\
3.82 \\
\mathrm{~mL} \cdot \mathrm{Kg}^{-} \\
\left.{ }^{1} \cdot \mathrm{min}^{-1}\right)\end{array}$ & $\begin{array}{l}\text { Pre: } \\
3 \times 30 \text { s WAnT with } 2- \\
\text { mins passive rest } \\
\text { between intervals }\end{array}$ & $\begin{array}{l}\text { Sport massage (SM) } \\
\text { AR (80rpm @ 40\% VO } \text { Vmax }) \\
\text { Passive lying in a supine } \\
\text { position (CON) } \\
\text { Duration: } \\
\text { 20-mins }\end{array}$ & BLa & $\begin{array}{l}\text { AR significantly } \downarrow \\
\text { BLa post-recovery } \\
\text { vs SM \& CON } \\
\text { (AR: }-59.38 \% \text {, } \\
\text { SM: }-36.21 \% \text {, } \\
\text { CON: }-38.67 \%) \\
\text { CON } \downarrow \text { BLa vs SM } \\
15 \text {-mins post } \\
\text { exercise }(p< \\
0.05) \text { but not at } 20 \\
\text { or } 25 \text {-mins }\end{array}$ & $\begin{array}{l}\text { AR > SM \& CON } \\
\downarrow \text { BLa } \\
\text { CON > SM } \downarrow \\
\text { BLa 15-mins } \\
\text { post exercise }\end{array}$ \\
\hline $\begin{array}{l}\text { Monedero } \\
\text { \& Donne, } \\
2000\end{array}$ & $\begin{array}{l}\mathrm{N}=10 \\
\text { Trained } \\
\text { male } \\
\text { cyclists } \\
\text { (age }=25 \pm \\
1 \text { years; } \\
\mathrm{VO}_{2 \max }=68 \\
\pm 1.7 \\
\mathrm{~mL} \cdot \mathrm{Kg}^{-} \\
1 \cdot \mathrm{min}^{-1} ; \\
\mathrm{PPO}=364 \\
\pm 9 \mathrm{~W} ; \\
\text { training } \\
\text { years }=5 \pm \\
0.3 \text { years) } \\
\mathrm{N}=18\end{array}$ & $\begin{array}{l}\text { Pre \& Post: } \\
5-\mathrm{km} \text { maximal effort } \\
\text { cycling test }\end{array}$ & $\begin{array}{l}\text { Passive seated at rest } \\
(\mathrm{CON}) \\
\text { AR }\left(50 \% \mathrm{VO}_{2 \max }\right) \\
\text { SM (lower leg) } \\
\text { Combined [AR \& SM] } \\
(3.75 \mathrm{~min} A R @ 50 \% \\
\text { VO }{ }_{2 \max } \text { pre and post-SM, } \\
7.5 \mathrm{~min} S \mathrm{SM}) \\
\\
\text { Duration: } \\
15 \text {-mins }\end{array}$ & $\begin{array}{l}\text { 5-km } \\
\text { performance } \\
\text { time } \\
\text { BLa } \\
\text { HR }_{\text {recovery }}\end{array}$ & $\begin{array}{l}\text { Combined } \\
\text { attenuated } \downarrow \\
\text { performance time } \\
\text { vs CON, AR \& SM } \\
\text { (performance time } \\
\text { increase between } \\
1^{\text {st }} \text { and } 2^{\text {nd }} \text { test; } \\
\text { CON: } 9.9 \pm 1.6 \\
\text { seconds, AR: } 6.9 \\
\pm 1.3 \text { seconds, } \\
\text { SM: } 7.7 \pm 1.5 \\
\text { seconds, } \\
\text { combined: } 2.9 \pm \\
1.5 \text { seconds, } \\
p<0.01 \text { ) } \\
\text { Combined } \downarrow \text { BLa } \\
\text { vs CON \& SM ( } p \\
<0.01 \text { ) } \\
\text { CON, SM \& SM } \\
\text { portion of } \\
\text { combined } \downarrow \\
\text { HRrecovery } \text { V AR \& } \\
\text { AR portion of } \\
\text { combined during } \\
\text { recovery ( } p< \\
0.05 \text { ) }\end{array}$ & $\begin{array}{l}\text { Combined > } \\
\text { CON, AR \& SM } \\
\text { attenuating } \downarrow \\
5 \mathrm{~km} \\
\text { performance } \\
\text { time } \\
\text { Combined \& AR } \\
>\text { CON \& SM } \downarrow \\
\text { BLa } \\
\text { CON \& SM > AR } \\
\downarrow \text { HRrecovery }\end{array}$ \\
\hline $\begin{array}{l}\text { Chan et } \\
\text { al, } 2016\end{array}$ & $\begin{array}{l}\text { Junior elite } \\
\text { male } \\
\text { cyclists } \\
\text { (age }=16 \pm \\
1 \text { year; } \\
\mathrm{VO}_{2 \mathrm{max}}= \\
64.7 \pm 4.3 \\
\mathrm{~mL} \cdot \mathrm{Kg}^{-} \\
{ }^{1} \cdot \mathrm{min}^{-1} \\
\mathrm{~N}=8\end{array}$ & $\begin{array}{l}\text { Pre: } \\
15 \text {-mins cycling @ } 75 \% \\
\text { PPO \& } 15-\text { min TT in heat } \\
\text { (TT1, } 31^{\circ} \mathrm{C}, 74 \% \text { rh) } \\
\text { Post: } \\
15 \text {-mins cycling @ } 75 \% \\
\text { PPO \& } 15-\text { min TT in heat } \\
\text { (TT2, } 31^{\circ} \mathrm{C}, 74 \% \text { rh) }\end{array}$ & $\begin{array}{l}\mathrm{CWI}\left(15^{\circ} \mathrm{C} \text {, mid-sternum }\right. \\
\text { level) } \\
\mathrm{CCT}\left(15^{\circ} \mathrm{C} \text {, ankle and }\right. \\
\text { thigh of both legs, rhythmic } \\
\text { compression setting HIGH) } \\
\text { AR @ } 40 \% \text { PPO }\left(31^{\circ} \mathrm{C}\right) \\
\text { Duration: } \\
10 \text {-mins passive seated in } \\
\left.\text { heat ( } 31^{\circ} \mathrm{C}, 74 \% \text { rh }\right) \\
15 \text {-mins per condition } \\
30 \text {-mins passive seated in } \\
\text { heat }\end{array}$ & $\begin{array}{l}\text { Mean power } \\
\text { Core body } \\
\text { temperature } \\
\text { BLa } \\
\text { RPE } \\
\text { HR }_{\text {recovery }}\end{array}$ & $\begin{array}{l}\text { No sig dif TT2 } \\
\text { mean power } \\
\text { between } \\
\text { conditions } \\
(p=0.551) \\
\text { CWI } \downarrow \text { core body } \\
\text { temperature 15- } \\
\text { mins during } \\
\text { recovery vs CCT } \\
(p=0.011) \\
\text { CWI } \downarrow \text { core body } \\
\text { temperature vs } \\
\text { AR post-recovery } \\
(p=0.033) \\
\text { AR } \downarrow \text { BLa vs CCT } \\
\& \text { CWI (AR: }-75 \%, \\
\text { CCT: -62\%, CWI: } \\
-62 \%) \\
\text { No sig dif RPE } \\
\text { between } \\
\text { conditions } \\
\text { No sig dif } \\
\text { HRrecovery between } \\
\text { conditions ( } p= \\
0.178)\end{array}$ & $\begin{array}{l}\mathrm{CCT}, \mathrm{CWI} \& \mathrm{AR} \\
=\text { mean power, } \\
\text { RPE \& HR Recovery } \\
\text { CWI > CCT } \downarrow \\
\text { core body } \\
\text { temperature post } \\
\text { treatment } \\
\text { CWI > AR } \downarrow \text { core } \\
\text { body } \\
\text { temperature } \\
\text { post-recovery } \\
\text { AR }>\text { CWI \& } \\
\text { CCT } \downarrow \text { BLa }\end{array}$ \\
\hline
\end{tabular}




\begin{tabular}{|c|c|c|c|c|c|c|}
\hline $\begin{array}{l}\text { Vaile } \\
\text { et al, } \\
2008 a\end{array}$ & $\begin{array}{l}\text { Well- } \\
\text { trained } \\
\text { male } \\
\text { cyclists } \\
\text { (age }=32 \pm \\
5 \text { years; } \\
\mathrm{VO}_{2 \max }= \\
70.7 \pm 7.9 \\
\mathrm{~mL} \cdot \mathrm{Kg}^{-} \\
{ }^{1} \cdot \mathrm{min}^{-1} \text { ) } \\
\mathrm{N}=10\end{array}$ & $\begin{array}{l}\text { Pre (Ex1): } \\
30-\text {-min cycling in heat }(34 \\
\pm 0.2^{\circ} \mathrm{C}, 39.4 \pm 1.5 \% \text { rh, } \\
15 \text {-min @ } 70 \% \text { PPO and } \\
\text { a } 15 \text {-min maximal cycling } \\
\text { TT) } \\
\text { Post (Ex } 2) \text { : } \\
30 \text {-min cycling in heat ( } 34 \\
\pm 0.2^{\circ} \mathrm{C}, 39.4 \pm 1.5 \% \text { rh, } \\
15 \text {-min @ } 70 \% \text { PPO and } \\
\text { a } 15-\text { min maximal cycling } \\
\text { TT) }\end{array}$ & $\begin{array}{l}\text { Shoulder height for all CWI } \\
\text { conditions } \\
\text { Intermittent CWI, } 10^{\circ} \mathrm{C} \\
\text { (ICWI10) } \\
\text { Intermittent CWI, } 15^{\circ} \mathrm{C} \\
\text { (ICWI15) } \\
\text { Intermittent CWI, } 20^{\circ} \mathrm{C} \\
\text { (ICWI20) } \\
\text { Continuous CWI, } 20^{\circ} \mathrm{C} \text {, in } \\
\text { bath for entire } 15-\text {-mins } \\
\text { (CCWI20) } \\
\text { AR (15-mins @ } 40 \% \\
\left.\text { VO2max, } 31.1 \pm 2.6^{\circ} \mathrm{C}\right) \\
\text { Duration: } \\
\text { Intermittent CWI }=5 \times 1 \text { - } \\
\text { min in bath, } 2 \text {-mins out of } \\
\text { bath }\left(29.2 \pm 1.4{ }^{\circ} \mathrm{C}, 58 \pm\right. \\
2.1 \% \text { rh) } \\
15 \text {-mins total per condition } \\
40-\text { mins passive recovery } \\
\left(34 \pm 0.2^{\circ} \mathrm{C}, 39.4 \pm 1.5 \%\right. \\
\text { rh) }\end{array}$ & $\begin{array}{l}\text { 30-min cycling } \\
\text { total work } \\
(\mathrm{kJ}) \\
\text { Body } \\
\text { temperature } \\
\text { BLa } \\
\text { RPE } \\
\text { HR }_{\text {post-intervention }} \\
\text { HR }_{\text {post-recovery }}\end{array}$ & 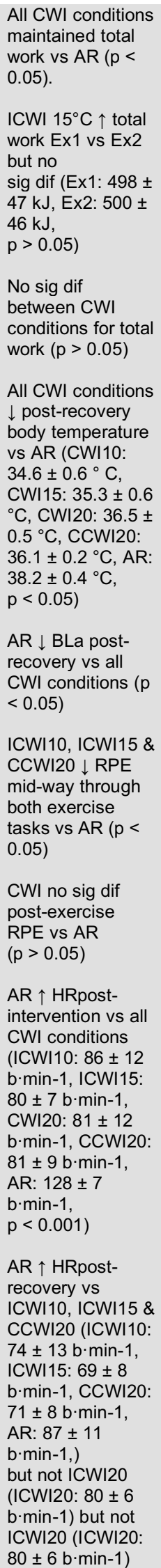 & $\begin{array}{l}\text { All CWI } \\
\text { conditions > AR } \\
\text { maintaining total } \\
\text { work and } \downarrow \text { post- } \\
\text { recovery body } \\
\text { temperature } \\
\text { AR > all CWI } \\
\text { conditions } \downarrow \text { BLa } \\
\text { ICWI10, ICWI15, } \\
\text { CCWI20 > AR } \downarrow \\
\text { RPE during } \\
\text { exercise } \\
\text { All CWI } \\
\text { conditions \& AR } \\
=\text { RPE post- } \\
\text { exercise } \\
\text { AR }>\text { all CWI } \\
\text { conditions } \uparrow \\
\text { HR post-intervention } \\
\text { AR > ICWI10, } \\
\text { ICWI15 \& } \\
\text { CCWI20 } \uparrow \\
\text { HR }\end{array}$ \\
\hline
\end{tabular}




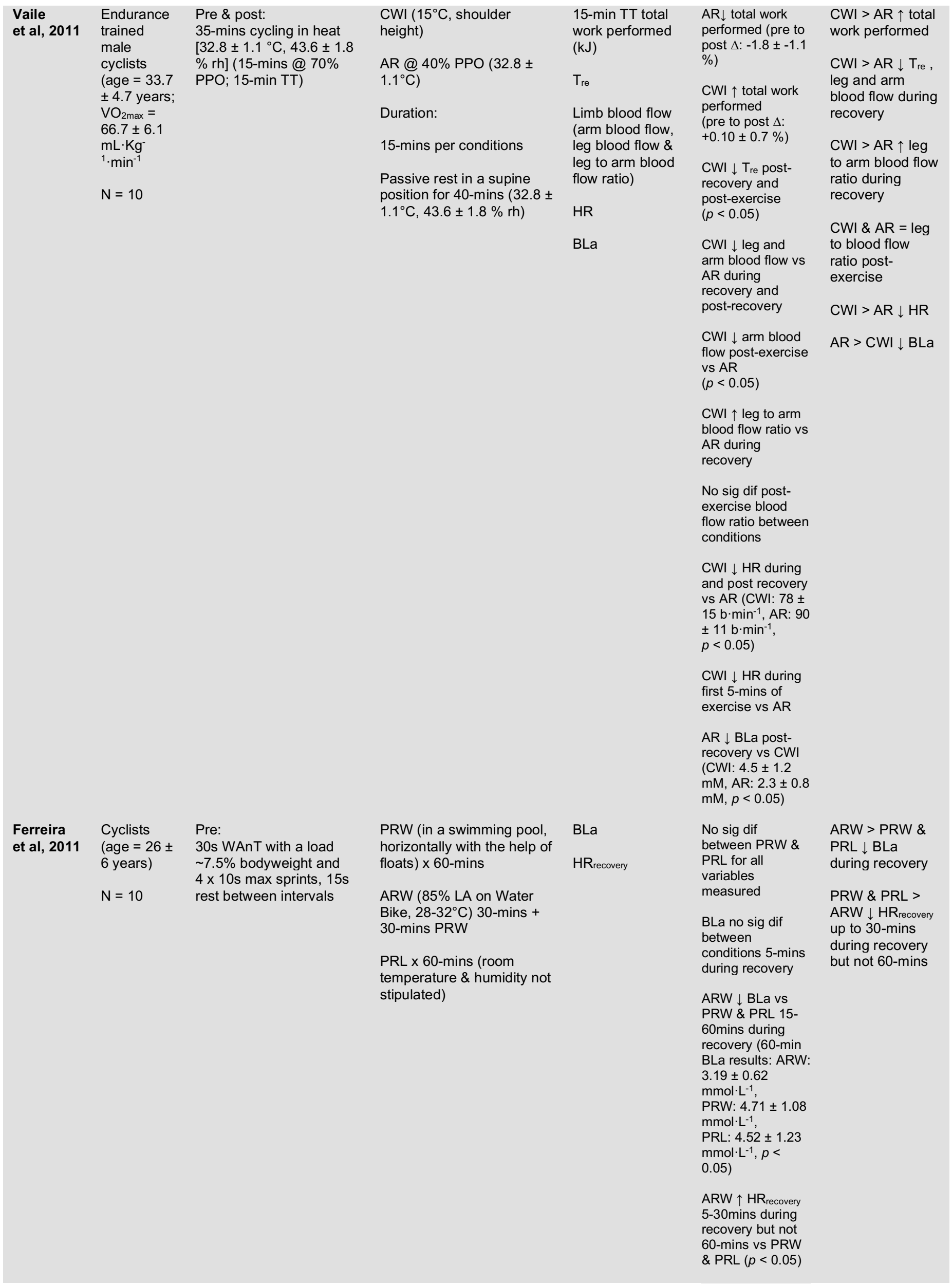

$N$ number of cyclists, $A R$ active recovery, CON control condition/passive rest, $P P O$ peak power output, $B L a$ blood lactate concentration, WAnT wingate anaerobic cycling test, SM sports massage, $H R$ heart rate, $V_{2 \max }$ maximal oxygen uptake, TT time trial, CWI cold water immersion, CCT cold compression therapy, RPE rating of perceived exertion, $r h$ relative humidity, $T_{r e}$ rectal temperature, $P R W$ passive recovery in water, $A R W$ active recovery in water, $P R L$ passive recovery on land. 


\section{Conclusions}

The use of COMP between 12 - 80mins post-exercise has been shown to improve subsequent 5 -min maximal cycling mean and max power output, 30s cycling mean power and 30-min cycling mean power (Argus et al. 2013; Chatard et al. 2004; Driller and Halson 2013; Ménétrier et al. 2013). CWI used for 5 -mins at $14^{\circ} \mathrm{C}$ following 25-mins of submaximal cycling has been shown to improve 4-km TT time to completion in the heat and average power output (Peiffer et al. 2008a). CWI used for $14-15 \mathrm{mins}$ at $15^{\circ} \mathrm{C}$ appears advantageous for improving 9-15min TT total work performed and repeated sprint power output (Vaile et al. 2008a; Vaile et al. 2008b; Vaile et al. 2011). CWI also appears more beneficial than AR at improving total work performed (Vaile et al. 2011). CWT used between 6-14mins with $38^{\circ} \mathrm{C}$ HWI and $15^{\circ} \mathrm{C} \mathrm{CWI}$ and a ratio of cold:hot 1:1mins or 1:2-mins, could increase subsequent TT total work performed, TT \& sprint mean power output and sprint PPO (Ménétrier et al. 2013; Vaile et al. 2008b). This performance benefit from CWT has been observed from durations as short as a $15 \mathrm{~s}$ sprint and up to a 15 min TT (Ménétrier et al. 2013; Vaile et al. 2008b). HWI alone appears to be detrimental to performance (Vaile et al. 2008b), while TWI has been shown to decrease 20$\mathrm{km}$ TT time to completion and improve average cycling speed (Lit et al. 2014). Both HUM and EMS may be able to attenuate the decrement in 30 s sprint mean power (Argus et al. 2013). SMOZO may assist time trial cycling performance (Paoli et al. 2013) and SM may improve anaerobic cycling mean power and reduce $5-\mathrm{km}$ TT time to completion (Bielik 2010; Monedero and Donne 2000). A combination of recovery strategies should be explored further, as AR and SM combined, were more beneficial than AR or SM alone, at reducing 5-km TT time to completion (Monedero and Donne 2000). The use of SS did not inhibit anaerobic cycling performance when performed for $3 \times 30$ s per muscle and leg (Kingsley et al. 2013) and may be a useful strategy for improving RoM and reducing the risk of knee injury when performed on the quadriceps muscle group, hamstrings muscle group and I.T.B between cycling exercise bouts (Asplund and St Pierre 2004). AR has been shown to attenuate $15 \mathrm{~s}$ sprint PPO, $5 \mathrm{~km}$ TT time to completion and even increase 30s sprint cycling mean power and PPO (Bielik 2010; Connolly et al. 2003; Monedero and Donne 2000).

A number of gaps exist in the current literature investigating the use of recovery techniques in cycling. Future research should aim to determine the influence of recovery strategies on multiple-day stage races (e.g. tours) and the also the influence of both CWI and HWI in different temperature environments. There is a paucity of research examining the use of recovery techniques in trained female cyclists and also the use of recovery strategies in a chronic (e.g. > 4 weeks) setting. Addressing these areas of future research will ensure a greater understanding of the use of recovery techniques and strategies in the sport of cycling.

\section{Conflict of Interest}

None

\section{References}

1. Abbiss C, Laursen P (2005) Models to explain fatigue during prolonged endurance cycling. Sports Medicine 35: $865-898$

2. Allen D, Lamb G, Westerblad H (2008) Skeletal muscle fatigue: cellular mechanisms. Physiological reviews 88: 287-332

3. Argus C, Driller M, Ebert T, Martin D, Halson S (2013) The effects of 4 different recovery strategies on repeat sprint-cycling performance. International Journal of Sports Physiology and Performance 8: 542-548

4. Asplund C, St Pierre P (2004) Knee pain and bicycling: fitting concepts for clinicians. The Physician and sportsmedicine 32: 23-30

5. Babault N, Cometti C, Maffiuletti N, Deley G (2011) Does electrical stimulation enhance post-exercise performance recovery? European Journal of Applied Physiology 111: 2501-2507

6. Bielik V (2010) Effect of different recovery modalities on anaerobic power in off-road cyclists. Biology of Sport 27: 59-63

7. Black M, Jones A, Blackwell J, Bailey S, Wylie L, McDonagh S, Thompson C, Kelly J, Sumners P, Mileva $\mathrm{K}$ (2017) Muscle metabolic and neuromuscular determinants of fatigue during cycling in different exercise intensity domains. Journal of Applied Physiology 122: 446-459

8. Brophy-Williams N, Driller M, Halson S, Fell J, Shing C (2014) Evaluating the kikuhime pressure monitor for use with sports compression clothing. Sports Engineering 17: 55-60

9. Brophy-Williams N, Driller M, Kitic C, Fell J, Halson S (2016) Effect of compression socks worn between repeated maximal running bouts. International Journal of Sports Physiology and Performance 12: 1-22

10. Buchheit M, Peiffer J, Abbiss C, Laursen P (2009) Effect of cold water immersion on postexercise parasympathetic reactivation. American Journal of Physiology-Heart and Circulatory Physiology 296: H421-H427

11. Cairns S (2006) Lactic acid and exercise performance. Sports Medicine 36: 279-291

12. Chan Y-Y, Yim Y-M, Bercades D, Cheng TT, Ngo K-L, Lo K-K (2016) Comparison of different cryotherapy recovery methods in elite junior cyclists. Asia-Pacific Journal of Sports Medicine, Arthroscopy, Rehabilitation and Technology 5: 17-23

13. Chatard J, Atlaoui D, Farjanel J, Louisy F, Rastel D, Guézennec C (2004) Elastic stockings, performance and leg pain recovery in 63-year-old sportsmen. European Journal of Applied Physiology 93: 347-352

14. Christensen P, Bangsbo J (2016) Influence of prior intense exercise and cold water immersion in recovery for performance and physiological response during subsequent exercise. Frontiers in Physiology 7: 1-10 15. Connolly D, Brennan K, Lauzon C (2003) Effects of active versus passive recovery on power output during repeated bouts of short term, high intensity exercise. Journal of Sports Science \& Medicine 2: 47-51 
16. Costa P, Ryan E, Herda T, Walter A, DeFreitas J, Stout J, Cramer J (2013) Acute effects of static stretching on peak torque and the hamstrings-toquadriceps conventional and functional ratios. Scandinavian Journal of Medicine \& Science in Sports 23: 38-45

17. Craig N, Norton K (2001) Characteristics of track cycling. Sports Medicine 31: 457-468

18. Cramer J (2008) Bioenergetics of exercise and training. Human Kinetics, Champaign, IL

19. Degroot M, Massie B, Boska M, Gober J, Miller R, Weiner M (1993) Dissociation of $[\mathrm{H}+]$ from fatigue in human muscle detected by high time resolution 31PNMR. Muscle \& nerve 16: 91-98

20. Driller M, Halson S (2013) The effects of lowerbody compression garments on recovery between exercise bouts in highly-trained cyclists. Journal of Science and Cycling 2: 45-50

21. Edwards B, Corte U (2010) Commercialization and lifestyle sport: lessons from 20 years of freestyle bmx in 'pro-town, USA'. Sport in Society 13: 1135-1151

22. Ferreira J, Da Silva Carvalho R, Barroso T, Szmuchrowski L, Śledziewski D (2011) Effect of different types of recovery on blood lactate removal after maximum exercise. Polish Journal of Sport and Tourism 18: $105-111$

23. Gardner S, Martin D, Jenkins D, Dyer I, Van Eiden J, Barras M, Martin J (2009) Velocity-specific fatigue: quantifying fatigue during variable velocity cycling. Medicine \& Science in Sports \& Exercise 41: 904-911 24. Halson S, Quod M, Martin D, Gardner A, Ebert T, Laursen P (2008) Physiological responses to cold water immersion following cycling in the heat. International Journal of Sports Physiology and Performance 3: 331346

25. Hasani A, Chapman T, McCool D, Smith R, Dilworth J, Agnew J (2008) Domiciliary humidification improves lung mucociliary clearance in patients with bronchiectasis. Chronic Respiratory Disease 5: 81-86

26. Jeukendrup A, Craig N, Hawley J (2000) The bioenergetics of world class cycling. Journal of Science and Medicine in Sport 3: 414-433

27. Jones A, Vanhatalo A, Burnley M, Morton R, Poole D (2010) Critical power: implications for determination of $\mathrm{VO}_{2 \max }$ and exercise tolerance. Medicine \& Science in Sports \& Exercise 42: 1876-1890

28. Kenney W, Wilmore J, Costill D (2015) Physiology of Sport and Exercise 6th Edition. Human Kinetics, Champaign, IL

29. Kingsley D, Zakrajsek R, Nesser T, Gage M (2013) The effect of motor imagery and static stretching on anaerobic performance in trained cyclists. The Journal of Strength \& Conditioning Research 27: 265-269

30. Lit K, Chen C, Ang B (2014) Effects of acute cool water immersion on time trial performance and exercised-induced oxidative stress among endurance cyclists in the heat. Journal of Athletic Enhancement 3: $1-5$

31. Marquet A-L, Hausswirth C, Hays A, Vettoretti F, Brisswalter J (2015) Comparison of between-trainingsessions recovery strategies for world-class bmx pilots.
International Journal of Sports Physiology and Performance 10: 219-223

32. Martin N, Zoeller R, Robertson R, Lephart S (1998) The comparative effects of sports massage, active recovery, and rest in promoting blood lactate clearance after supramaximal leg exercise. Journal of Athletic Training 33: 30-35

33. Mena P, Maynar M, Campillo J (1996) Changes in plasma enzyme activities in professional racing cyclists. British Journal of Sports Medicine 30: 122-124

34. Ménétrier A, Pinot J, Mourot L, Grappe F, Bouhaddi M, Regnard J, Tordi N (2013) Effects of recovery using contrast water therapy or compression stockings on subsequent 5-min cycling performance. Journal of Science and Cycling 2: 49-56

35. Millet G, Lepers R (2004) Alterations of neuromuscular function after prolonged running, cycling and skiing exercises. Sports Medicine 34: 105116

36. Monedero J, Donne B (2000) Effect of recovery interventions on lactate removal and subsequent performance. International Journal of Sports Medicine 21: 593-597

37. Nédélec M, McCall A, Carling C, Legall F, Berthoin S, Dupont G (2013) Recovery in soccer. Sports Medicine 43: 9-22

38. Overmayer R, Driller M (2018).

39. Paoli A, Bianco A, Battaglia G, Bellafiore M, Grainer A, Marcolin G, Cardoso CC, Dall'Aglio R, Palma A (2013) Sports massage with ozonised oil or non-ozonised oil: comparative effects on recovery parameters after maximal effort in cyclists. Physical Therapy in Sport 14: 240-245

40. Peiffer J, Abbiss C, Nosaka K, Peake J, Laursen P (2007) Effect of cold water immersion after exercise in the heat on muscle function, body temperatures, and vessel diameter. Journal of Science and Medicine in Sport 12: 91-96

41. Peiffer J, Abbiss C, Watson G, Nosaka K, Laursen P (2008a) Effect of a 5-min cold-water immersion recovery on exercise performance in the heat. British Journal of Sports Medicine 44: 461-465

42. Peiffer J, Abbiss C, Watson G, Nosaka K, Laursen P (2008b) Effect of cold water immersion on repeated 1$\mathrm{km}$ cycling performance in the heat. Journal of Science and Medicine in Sport 13: 112-116

43. Peiffer J, Abbiss C, Watson G, Nosaka K, Laursen P (2009) Effect of cold-water immersion duration on body temperature and muscle function. Journal of Sports Sciences 27: 987-993

44. Phillips S (2015) Fatigue in sport and exercise. Routledge, New York, NY

45. Robergs R, Ghiasvand F, Parker D (2004) Biochemistry of exercise-induced metabolic acidosis. American Journal of Physiology-Regulatory, Integrative and Comparative Physiology 287: R502-R516

46. Samuel M, Holcomb W, Guadagnoli M, Rubley M, Wallmann H (2008) Acute effects of static and ballistic stretching on measures of strength and power. The Journal of Strength \& Conditioning Research 22: 14221428 
47. Schniepp J, Campbell T, Powell K, Pincivero D (2002) The effects of cold-water immersion on power output and heart rate in elite cyclists. The Journal of Strength \& Conditioning Research 16: 561-566

48. Stanley J, Buchheit M, Peake J (2012) The effect of post-exercise hydrotherapy on subsequent exercise performance and heart rate variability. European Journal of Applied Physiology 112: 951-961

49. Stanley J, Peake J, Buchheit M (2013) Consecutive days of cold water immersion: effects on cycling performance and heart rate variability. European Journal of Applied Physiology 113: 371-384

50. Temesi J, Mattioni-Maturana F, Peyrard A, Piucco T, Murias J, Millet G (2017) The relationship between oxygen uptake kinetics and neuromuscular fatigue in high-intensity cycling exercise. European Journal of Applied Physiology 117: 969-978

51. Vaile J, Halson S, Gill N, Dawson B (2008a) Effect of cold water immersion on repeat cycling performance and thermoregulation in the heat. Journal of Sport Science 26: 431-440

52. Vaile J, Halson S, Gill N, Dawson B (2008b) Effect of hydrotherapy on recovery from fatigue. International Journal of Sports Medicine 29: 539-544

53. Vaile J, O'Hagan C, Stefanovic B, Walker M, Gill N, Askew C (2011) Effect of cold water immersion on repeated cycling performance and limb blood flow. British Journal of Sports Medicine 45: 825-829

54. Versey N, Halson S, Dawson B (2011) Effect of contrast water therapy duration on recovery of cycling performance: a dose-response study. European Journal of Applied Physiology 111: 37-46

55. Westerblad H, Allen D, Lännergren J (2002) Muscle fatigue: lactic acid or inorganic phosphate the major cause? American Physiological Society 17: 17-21 